\title{
Unstructured Grids for Sonic Boom Analysis and Design
}

\author{
Richard L. Campbell ${ }^{1}$ \\ NASA Langley Research Center, Hampton VA 23681 \\ and \\ Sudheer N. Nayani ${ }^{2}$ \\ Analytical Services \& Materials, Inc., Hampton, VA 2366
}

\begin{abstract}
An evaluation of two methods for improving the process for generating unstructured CFD grids for sonic boom analysis and design has been conducted. The process involves two steps: the generation of an inner core grid using a conventional unstructured grid generator such as VGRID, followed by the extrusion of a sheared and stretched collar grid through the outer boundary of the core grid. The first method evaluated, known as COB, automatically creates a cylindrical outer boundary definition for use in VGRID that makes the extrusion process more robust. The second method, BG, generates the collar grid by extrusion in a very efficient manner. Parametric studies have been carried out and new options evaluated for each of these codes with the goal of establishing guidelines for best practices for maintaining boom signature accuracy with as small a grid as possible. In addition, a preliminary investigation examining the use of the CDISC design method for reducing sonic boom utilizing these grids was conducted, with initial results confirming the feasibility of a new remote design approach.
\end{abstract}

\section{Nomenclature}

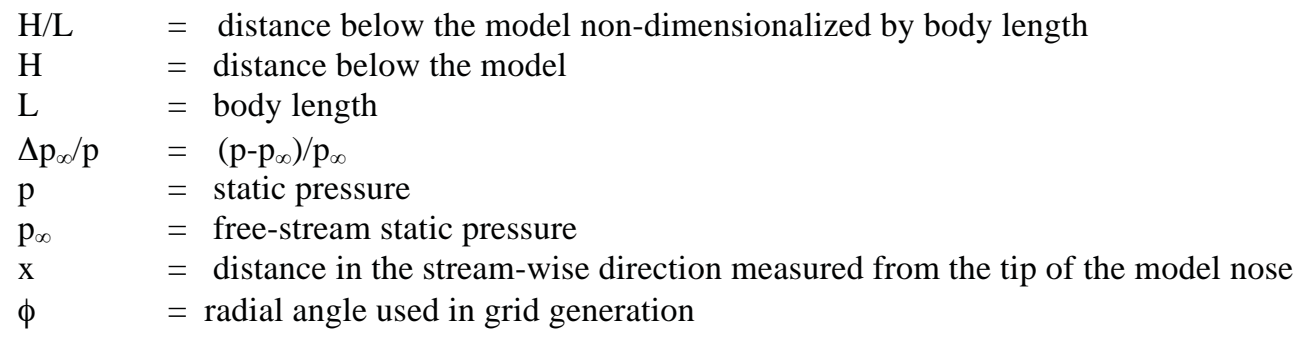

\section{Introduction}

The lure of cutting travel times in half relative to current transport aircraft continues to spark interest in developing a viable supersonic transport. Over the last decade, NASA and industry have worked together to identify and address some of the technical challenges to this viability. One key challenge that is being addressed is the goal of reducing the sonic boom to levels that would allow supersonic flight over land while maintaining efficient aerodynamic performance. Although there is currently no approved boom level for supersonic flight over the US and most

\footnotetext{
${ }^{1}$ Senior Research Engineer, Configuration Aerodynamics Branch, MS 499, AIAA Associate Fellow.

${ }^{2}$ Senior Scientist, CFD Group, 107 Research Drive, AIAA Senior Member.
} 
countries, a target level of 85 PLdB (perceived decibel level) has been proposed by NASA for the purpose of focusing some of the design activities within the N+2 Supersonic Validation studies as part of the Supersonic Project in the NASA Fundamental Aerodynamics Program ${ }^{1}$.

Computational fluid dynamics (CFD) analysis and design tools have become increasingly important in the development of configurations that approach the desired boom levels, with data for evaluation coming from flight and wind tunnel tests. Two flight experiments that provided such data and validated concepts for reducing the contribution to boom levels from the front of an aircraft are the tests of the Shaped Sonic Boom Demonstration (SSBD) aircraft ${ }^{2}$ and the Quiet Spike concept test series ${ }^{3}$. More recently, Boeing ${ }^{4}$ and Lockheed ${ }^{5}$ developed configurations under the $\mathrm{N}+2$ studies mentioned above, addressing the boom signature for the whole aircraft as well as trying to keep drag levels low. Wind tunnel models of these configurations were built and tested in the 9- by 7Foot Supersonic Wind Tunnel at NASA Ames Research Center ${ }^{6-8}$.

Data from these as well as previous tests of other configurations formed the basis for the $1^{\text {st }}$ AIAA Sonic Boom Prediction Workshop held in 2014 and other research on sonic boom prediction. An excellent summary of the Workshop results is given by Park ${ }^{9}$. While there were some variations in the comparisons between wind tunnel data and CFD results for the different flow solvers, overall the results indicated reasonable correlation and a significant improvement over the predictive capabilities from a decade ago. Much of this improvement is due to advances in the generation of CFD grids for sonic boom analysis, including both static grid generation as well as grid adaptation methods. In a recent paper $^{10}$, three static methods that utilize grid extrusion techniques (BG, MCAP and INFLATE) are compared, with all three showing good accuracy relative to wind tunnel data.

The purpose of the current paper is to document some extensions to one of these methods, the COB/BG system, that are intended to make the process easier to use as well as more accurate and efficient. In addition, parametric studies were performed to develop some best practice guidelines for its use. While this study used a specific core grid generation code and flow solver from the TetrUSS software system ${ }^{11}$, it is hoped that the guidelines may be applicable to other codes as well. Finally, the use of these grids in conjunction with the CDISC design method will be assessed for sonic boom design.

\section{Grid Generation Process}

The grid generation process in this study uses the GRIDTOOL, VGRID and POSTGRID codes from the TetrUSS software system. GRIDTOOL is a graphics-based pre-processor that is used to build an input file for VGRID from an IGES definition of the model. The VGRID code utilizes advancing layer and/or advancing front grid generation methods to triangulate the model surface and fill the grid volume with tetrahedral cells. The POSTGRID code is then used to close any voids in the grid as well as improve grid quality. In conjunction with this software, the AUTOSRC ${ }^{12}, \mathrm{COB}^{10}$ and $\mathrm{BG}$ codes $^{13}$ developed by the authors were used to facilitate the process and create the full boom grid. The next two sections will describe the COB and BG codes in more detail and give some suggestions for their use based on results from parametric studies.

The overall process consists of two primary steps: 1) the generation of an inner "core" grid around the model; and 2) the extrusion of a stretched and sheared "collar" grid from the core grid outer boundary. In the first step, GRIDTOOL was used to develop a set of points, curves and patches that define the half-model geometry as well as an initial symmetry plane. Based on this geometry, the AUTOSRC code automatically determines the initial size and location of line and volume sources required by VGRID to achieve the desired resolution for the model surface grid, with minor additional manual refinement used in some cases. The COB code is then used to create the remaining outer boundary geometry definition and associated line sources that provide the desired resolution for the collar grid. The resulting input file is fed into VGRID and POSTGRID to generate the core grid.

Once the core grid is generated, the second step of the process, the generation of the collar grid around the core grid using BG, can be completed. This typically takes less than a minute, compared to generating the core grid, which can take 1-2 orders of magnitude longer. As the COB and BG codes have a primary impact on the size and accuracy of the final boom grid, these are discussed below, noting in particular capabilities added since the previous study. 


\section{Cylindrical Outer Boundary (COB) Code}

From previous work ${ }^{13}$, the primary factors determining the robustness, accuracy and efficiency (in terms of grid size) for sonic boom calculations made on a grid from an extrusion method such as BG are the location of the outer boundary relative to the bottom of the aircraft and the grid spacing on that boundary. The cylindrical outer boundary (COB) code was developed to automatically generate the points, curves, patches and sources required by VGRID for an outer boundary that meets spacing and location requirements, with compatible inflow, outflow and symmetry planes also defined.

The key feature in COB that affects the robustness of the collar grid in BG is the definition of multiple patches around the circumference of the outer boundary and associated line sources along the stream-wise edges (see fig 1), with spacing defined to provide a single triangle across each patch. As addressed in Park ${ }^{10}$ and Ordaz ${ }^{14}$, this guarantees positive cell volumes during the radial extrusion process used in the BG code. The dense array of line sources also eliminates the volume source that was previously required under the aircraft to provide adequate axial and circumferential spacing for propagating boom signature details.

In general, the aspect of the core grid that affects the accuracy of the solution on the final BG grid is the face size on the outer boundary. In order to provide the optimum accuracy for a given total number of grid cells, several options are included in $\mathrm{COB}$ to vary grid spacing in both the axial and circumferential directions. The new version provides up to twenty axial regions with a specified spacing at each end, allowing the user, for example, to use coarser spacing ahead of and behind the aircraft, medium spacing for most of the body and a fine grid under the nacelle region. In similar fashion, the circumferential spacing is typically increased going from the bottom to the top of the outer boundary to reduce the total number of cells. For off-track calculations, the finer spacing directly under the aircraft can be maintained to a desired circumferential angle to improve resolution, but at the cost of more collar grid cells.

One option that has a positive effect on signature accuracy while possibly reducing grid size is to locate the bottom of the aircraft as close to the core grid outer boundary as possible. This minimizes the region of un-stretched, unsheared cells that the signature has to travel through that would tend to dissipate the signal. Adding a volume source under the aircraft if it is placed closer to the center of the core grid could help reduce some of the dissipation, but at the cost of more cells. The $\mathrm{COB}$ code input includes configuration geometry information that is used to automatically size and locate the outer cylinder for a close fit under the aircraft. This benefit will be illustrated in a later section of the paper.

An additional capability recently added to the $\mathrm{COB}$ code is the generation of a rectangular outer boundary (ROB) VGRID input file. With this option, the lower boundary is located very close to the bottom of the fuselage and the side and top walls positioned far enough from a configuration (typically one body length) to avoid contamination of the boom signature from any wave reflections. This option required a corresponding change in the BG code to allow extrusion of the collar grid only through the lower face, with the goal of requiring fewer grid cells for the same boom signature accuracy. For this option, a single patch is defined for the lower boundary, with multiple line sources used to control the face sizes on this patch similar to the cylindrical outer boundary method (figure 2). While this approach doesn't guarantee the elimination of negative cells in the generation of the collar grid, initial experience indicates that the flat extrusion patch surface delays the creation of negative cells to distances well beyond the typical (3-10 body lengths) values used. A preliminary evaluation of this approach will be included in the Results section of this paper.

\section{Boom Grid (BG) Code}

After the outer boundary input is defined and added to the VGRID input file by COB, the core grid is generated and passed to the BG code. This code uses a process that extrudes prisms through core grid outer boundary faces at a radial angle from a given reference point (see figure 3), then splits each prism into three tetrahedral cells using an efficient algorithm that ensures matching of adjacent faces. A method of moving the reference point during the grid 
generation process allows the clustering of grids at an off-track angle for better resolution of signatures at that location (see figure 4). More details of this method and its basic capabilities are given in Nayani ${ }^{13}$.

Since the number of extrusion faces in the collar grid is determined from the core grid, the only controls in the original BG code on the total number of cells in the collar grid are the radial stretching factor and the radial extent of the final outer boundary. Although they do not change the final grid size, the adjustment to the shearing angle and off-track clustering capabilities can affect the accuracy of the computed boom signatures. Results from parametric studies of these parameters will be included in a subsequent section.

In an effort to further reduce the number of grid cells needed to obtain an accurate boom signature, an option was added to BG to extrude cells only through selected patches on the core grid outer boundary. This capability, particularly when used with the ROB option described in the section above, can have a large impact on the number of cells in the collar grid, which for Euler core grids and/or large signature distances could be a major percentage of the total number of grid cells.

One additional feature that has been included in BG with possible application to design on these grids is the capability to generate initial collar grid layers without stretching or shearing. For solution accuracy, the core grid is usually positioned very close to the lower surface of the model. If design changes are large enough, the volume grid modification process that allows use of flow solution restarts for efficiency may require changes to the collar grid. Because of the high aspect ratio of these cells, this may produce distorted cells that can affect propagation or negative volume cells that can prevent the flow solver from running. As long as the number of un-sheared initial cell layers is small, the maximum stretching ratio can be increased slightly to reach the same signature location with the original number of total layers, thus allowing a direct solution restart. Alternatively, the stretching ratio can be held constant, requiring more total layers but at least avoiding the generation of a new core grid.

\section{Flow Analysis and Design}

The USM3D flow solver, also part of the TetrUSS software system, was used for all of the flow analysis and design cases shown in this paper. It is a cell-centered, finite-volume Navier-Stokes flow solver that uses Roe fluxdifference splitting ${ }^{15}$ to compute inviscid flux quantities across the faces of the tetrahedral cells. The minmod limiter was used to improve solution robustness for all cases. Computations were made on inviscid and viscous grids, depending on the configuration, with the viscous grid solutions using Mentor's shear stress transport (SST) turbulence model ${ }^{16}$. Because the number of flow variables in USM3D is related to the number of cells, this is the value that will be quoted with reference to grid sizes.

For the part of the study related to design, the CDISC design method ${ }^{17}$ was used in conjunction with USM3D. The CDISC method uses a knowledge-based approach with prescribed sensitivity derivatives developed from smalldisturbance flow approximations. This allows the flow solution and design to converge in parallel, which greatly reduces design times relative to formal numerical optimization approaches. A number of flow constraints are available to automatically define target pressure distributions based on desired flow characteristics such as low wave $\mathrm{drag}^{17}$ or increased extent of laminar flow ${ }^{18}$, or a user-prescribed target pressure may be input. Geometry constraints are also available to meet requirements related to manufacturing, structures, etc.

For typical applications of CDISC, the aircraft surface geometry is changed to drive its pressure distribution to a desired target pressure distribution on that surface. This approach could be applied to design for reduced sonic boom as well, cleaning up local flow disturbances at the surface that propagate into the near field as shocks and expansions that add to the noise level of the signature. While this approach is direct in the sense that it tries to eliminate unwanted features at their source, it does not take into account the interaction of the features as they propagate, such as merging or cancelling.

In order to apply CDISC to a remote target pressure such as a near-field sonic boom signature, it is necessary to relate geometry changes on the aircraft surface to changes in the analysis pressures at the remote location. The nearfield target (NFTARG) code was written to do this. It employs a ray-tracing technique to track the influence of a point on the keel line of the fuselage into the field. A marching approach is applied, with the local Mach number of the nearest point on the symmetry plane grid used to determine the angle of the next marching step. This process 
continues until the signature location below the model is reached. To help minimize any inaccuracies in this approach, the signature is shifted to align with the nose point ray trace line if needed. This approach had been successfully used with the Cart3D flow solver ${ }^{19}$ in some previous unpublished work. With that method, however, the symmetry plane grid was very uniform and the associated grid faces had stretching ratios on the order of 8-10. For the current work, the initial unstructured grid is far less uniform and may get distorted further by the grid movement in the design process. This, along with the higher grid stretching ratios of 50 or more involved, raises the question of the accuracy of interpolating Mach numbers from the nearest grid point.

In theory, this approach could be applied to the design of any aircraft component for on- or off-track targets. To simplify the evaluation of the concept, the included example is limited to fuselage design to match an on-track boom signature target. For this case, the basic CDISC algorithm remained unchanged, but the sensitivity derivatives were scaled to account for the decay in the pressure signal due to propagation to the remote location.

\section{Results}

The analysis and design results presented in this study were obtained using the three configurations from the $1^{\text {st }}$ AIAA Sonic Boom Prediction Workshop. These include a body of revolution (SEEB), a delta-wing/body configuration (DWB), and a low-boom supersonic transport concept (LM1021). Descriptions of the models and selected experimental results from wind tunnel tests of them are given in Cliff ${ }^{8}$. Images of the models used in the CFD calculations are shown in figure 5. Note that all are half models, so only the left half of the center-line nacelle over the fuselage on the LM1021 is visible, along with the left half of the blade sting extending from the top of the fuselage. The LM1021 geometry definition had been rotated to an angle of attack of 2.1 degrees to reflect the test condition used in the cases shown below, thus no angle of attack adjustment was used in USM3D or BG.

\section{COB Evaluations}

The DWB configuration was used to evaluate several of the parameters used in COB that can impact the accuracy. All of the solutions shown for the DWB cases were run using USM3D in Euler mode at a Mach number of 1.7 and angle of attack of 0.0 degrees, consistent with the test case in the $1^{\text {st }}$ AIAA Sonic Boom Prediction Workshop.

The first parameter investigated is the vertical location of the model in the core grid. The left side of figure 6 shows a portion of the symmetry plane grid for the model placed in the center of the un-sheared core grid, with the right side showing a similar picture of the grid with the configuration automatically located near the bottom of the core grid based on geometry information input to COB. Restrictions on core grid diameter and how close the wing tip can be to the core grid boundary prevent the core grid from being any closer to the bottom of the fuselage. Figure 7 shows the on-track ( $\phi=0$ degrees) boom signatures cut at 24.86 inches (3.6 body lengths) below the nose for each of these cases. While the general features are captured by both approaches, the lower fuselage position produces less dissipation as indicated by the higher peak values.

The second parameter input to COB that can influence the accuracy of the signature is the axial spacing for the core grid outer boundary. Five core grids were generated using axial grid spacing determined by the body length divided by 100, 200, 300, 400 and 500. Thus, the 100 grid would have the coarsest axial spacing and the 500 grid the finest. Boom grids were then created for each of these core grids, with the final grids having 2, 5, 11, 22 and 40 million cells, respectively. The resulting near-field signatures at approximately 3.6 body lengths below the nose for track angles of 0,30 and 60 degrees are shown in figure 8, with a close-up of the large expansion just past $\mathrm{x}=1$ included to the right of each full signature plot. The differences between the curves for a spacing of 300 or greater are barely perceptible. For the 100 and 200 cases, the signatures are still similar, but the peaks are noticeably reduced and shifted, with the differences increasing with off-track angle. The latter effect may be due in part to the circumferential spacing being related to the axial spacing. These results indicate that the axial spacing factor of 300 is probably adequate and would significantly reduce grid size relative to the 400 or 500 cases, which would be especially important for design applications.

A new option in COB is the ability to retain the dense spacing at the bottom of the core grid outer boundary around the circumference to a user-specified off-track angle for cases involving off-track signatures. To evaluate this option, the 300 axial spacing factor from the case above was used to generate two more grids that retained the dense spacing 
to 30- and 60-degree off-track angles. In addition to the denser spacing along the core grid outer boundary for the latter two cases, the option in BG to focus the grid at the desired off-track angle was utilized when generating the collar grids. The final grids had 17 and 24 million cells, respectively, compared to the on-track grid with 11 million cells. Signatures from analyses on these grids are shown in figure 9. Close up views of the signature peak and valley regions (not shown) indicate differences in level of less than 0.0005 , thus there is no significant benefit to retaining the dense grid spacing to off-track angles, especially in light of the penalty in grid size.

As previous studies with $\mathrm{BG}^{13}$ had indicated some improvement in signature accuracy at off-track angles with the grid focusing option, it raises the possibility that the DWB configuration, being a simple wing-body model, is perhaps not very sensitive to grid differences. To address this question, a similar study was made with the LM1021 configuration. Two grids were generated, one for on-track and one for 50-degrees off-track, with both the COB and BG off-track options applied to the second case. The grids were for viscous mode in USM3D and consisted of 37 and 54 million cells for the two cases, respectively. The cases were run at a Mach number of 1.6, angle of attack of zero degrees and a Reynolds number of 1.4 million based on mean aerodynamic chord of 6.628 inches. A comparison of the signatures from the two grids at track angles of 0,30 and 50 degrees is shown in figure 10. As with the DWB case, the signatures are nearly identical at each track angle. Apparently, the relatively slow growth rate in circumferential spacing around the outer boundary supplied by the COB patching and sourcing scheme required to avoid negative cells in BG is adequate to resolve off-track signatures and no additional refinement is needed.

In general, $\mathrm{COB}$ grids have been generated using three axial spacing zones. These consist of a fine spacing extending from just ahead of the nose to sufficiently far behind the aircraft to pick up any propagated signals. For example, the aft extent of the fine grid must be extended if there is a vertical tail on the configuration. The spacing is then linearly increased to the inflow and outflow planes to a value typically about four times as large as the center zone spacing in order to reduce the total number of grid cells. In response to a user request, the number of spacing regions has been increased to twenty to allow locally denser regions under the body (e.g., to better resolve flow features from the nacelle).

To demonstrate this new option, two grids were generated about the DWB configuration, one with the previous approach using three regions and a second grid using seven spacing regions. The axial distribution of spacing along the bottom of the core grid outer boundary for the two grids is shown in figure 11. The fuselage extends from 0 . to 0.175 , with the wing root leading and trailing edges at 0.082 and 0.163 , respectively. In this example, the extra zones were used to retain the densest grid underneath the wing only, thus reducing the number of cells relative to the original approach, which retained this level of grid spacing underneath the entire body. After generating collar grids for each case with BG, the resulting grid sizes were 18.1 million cells for the original approach and 10.8 million cells for the new approach. The resulting signatures for each grid are plotted in figure 12 and indicate no significant penalty in peak magnitudes in the regions of reduced spacing. This was surprising in that the corresponding spacing parameter (175) is about half of what would have been recommended based on previous results.

The final new capability in COB evaluated in this study is the rectangular outer boundary (ROB) option. Two grids were created for the DWB configuration using this approach, one (ROB) with a horizontal lower outer boundary patch and a second with the lower boundary rotated about its intersection with the symmetry plane (ROBT) so that it is the same distance under the wing tip as under the fuselage at the symmetry plane. The reason for the tilted lower boundary in the second grid is to bring the beginning of the sheared grid closer to the wing to reduce any signal dissipation from traversing the un-sheared region of the grid. The collar grid inflow planes for these two grids are shown in figure 13, along with the inflow plane from a $\mathrm{COB}$ grid with the same axial spacing at the symmetry plane.

The respective sizes for the COB, ROB and ROBT grids are 18.1, 4.9, 4.7 million cells. The significant reduction in the number of grid cells for the latter two grids relative to the cylindrical one is the result of a couple of factors. The first is obvious from figure 13, in that, even though all three grids have the same off-track spacing (circumferential for $\mathrm{COB}$ and span-wise for $\mathrm{ROB}$ ) under the fuselage, the ROB approach does not require that this spacing be maintained to the inflow (or outflow) planes. The patching scheme used in COB to avoid negative cells in BG, however, does force the circumferential boundary face sizes to be the same for the whole length of the grid. The second reason for smaller grids is that fewer off-track faces are needed, as the collar grid is only extruded through the lower boundary instead of the full outer boundary in BG for the ROB option. One additional minor benefit to the 
ROB approach relative to COB is that it speeds up both the VGRID and BG grid generation tasks because of fewer patches and sources to process.

The on-track signatures for the above three cases are shown in figure 14 and are nearly identical, with no real benefit seen for the tilted lower boundary (ROBT). Studies are underway to assess the off-track accuracy for the rectangular grids. Currently, the span-wise distribution of spacing is controlled by an overall growth factor (increasing spanwise), but other options such as clustering at given off-track angles are being considered. A preliminary check on the grid focusing capability in BG indicates that this option works for these grids as well as the original cylindrical ones.

\section{BG Evaluations}

For the BG code, there are two input parameters that can impact the number of layers added in the collar grid and thus the total number of cells: the maximum stretching factor, and the radial extent of the grid in body lengths. In growing the collar grid in the BG code, the height of the first layer of cells is determined from the average axial spacing at the bottom of the core grid outer boundary times a growth factor, which has been hardwired to 2.0 based on previous studies. This growth factor is then applied to subsequent layers until the layer spacing reaches the maximum stretching limit. Prior work suggested that a limit of 50 times the initial spacing gave good results, so this value was set to correspond to a stretching factor of 1.0 to simplify the input. A higher stretching factor requires less layers to reach a given signature location, but the increased stretching could result in code accuracy or stability problems.

To evaluate the effects of maximum stretching factor, four grids were generated using factors of $0.25,0.5,1.0$ and 2.0 to reach an outer boundary location of five body lengths from the nose of the model. The sizes for these grids were 43, 26, 17 and 13 million cells, respectively. This confirms the robustness that the COB method brings to the boom grid process, as the BG code had failed to generate grids to the desired radial distance for the lower two maximum stretching factors when the previous method for defining the core grid outer boundary was used. A plot of the on-track signature at 3.6 body lengths below the aircraft is shown in figure 15 . The signatures for all four cases are nearly identical, with maximum differences of less than 0.001 in peak height. As shown in the enlargement of the peak near $x=0.96$ (figure 15b), the peak height decreases slightly with increasing maximum stretching factor, indicating some increased dissipation. Based on the cost in grid size for the small improvement in accuracy, a stretching factor of 1.0 would still seem to be the best choice. The ultimate impact of differences in near-field signatures such as these would have to be assessed by propagation to ground level. However, as was shown in Park ${ }^{9}$, variations in near-field signature levels that were an order of magnitude larger than those shown in figure 15 produced less than 1 PLdB variation on the ground, so the effects here should be minimal.

The radial extent of the grid obviously must exceed the near-field boom signature location. In the example above, it was assumed that a cushion of one body length or more was required to avoid any contamination from features possibly reflected from the outer boundary. In theory, the characteristic boundary condition used there should allow waves to pass through that grid surface but this had not been confirmed. To test this assumption, two grids were created using BG from the same core grid about the DWB configuration: one with the signature location within the outermost layer of cells $(\mathrm{R}=3.6$ in figure 16), and one with addition layers of cells extending the boundary another half body length $(\mathrm{R}=4.1)$. The figure shows that there is no significant difference in the two signatures and that, at least at this signature distance, which is representative of that used for propagation-to-ground studies, the outer boundary may be placed just beyond the signature location.

One BG input parameter that does not affect grid size but can have a very important impact on signature accuracy is angle of attack. If the flow solver is run at a non-zero angle of attack, this angle should be used in BG as well to ensure that the grid shearing is properly aligned. Park ${ }^{10}$ illustrates the effects of grid misalignment using a simple 2$\mathrm{D}$ example of flow over a double ramp. To evaluate the effect on a more realistic configuration, grids were created for the LM1021 configuration using BG with alpha off-sets of -4, -2, 0, 2 and 4 degrees. Note that with this approach, the original core grid could be used for each of these cases. For each case, the flow solver was run at a Mach number of 1.6, angle of attack of 0.0 degrees and a Reynolds number of 1.4 million. The resulting on-track signatures at 31.8 inches ( 1.4 body lengths) below the model nose are shown in figure 17 for alpha $=-2,0$ and 2 degrees ( -4 and 4 degrees were omitted for clarity). For both non-zero alpha cases, the initial peak is washed out 
completely, the large peak near $\mathrm{x}=4.7$ is greatly reduced and the other smaller peaks are significantly damped. Consistent with Park's case, the effect is smaller for the negative angle case. If the angle offsets are converted to an equivalent Mach number based on the free-stream Mach number of 1.6, values of 1.674 and 1.534 are obtained for alpha $=-2$ and 2, respectively. These Mach levels are typically found only very close to the model and would obviously not match characteristic directions further into the field.

\section{Design Evaluations}

Design for reduction of sonic boom has a number of requirements related to grids that differ from the more typical use of the CDISC method for drag reduction. First, the grids are considerably larger for boom design due to the need for dense grid in the field to accurately propagate the signature to the desired location. This was a key motivator for the parametric studies described in the sections above, especially in view of multiple solutions being required for design. Another distinction between design for boom and drag is that more flow solver iterations are needed per design cycle for boom design to allow time for the changes to propagate to the signature location. Smaller grids in general can reduce the time required but, in particular, using larger maximum stretching factors give fewer cells between the model and the signature location and should keep the extra cycle penalty to a minimum.

One additional grid-related difference for boom design concerns the movement of the volume grid to absorb design changes to the surface grid. A contributor to the efficiency of the CDISC approach is the use of flow solver restarts, which requires the same number of points as well as face and cell connectivity for each design cycle. For a grid of good quality (e.g., very few "sliver" cells with very small vertex angles), the CDISC process generally works well and, if negative volume cells are created, they can typically be corrected. The BG code purposely creates highlystretched cells in the collar grid, so if the design changes are large enough, they may affect these cells and be difficult to fix or result in distorted cells that affect the accuracy.

As noted earlier, there is an option in BG to generate a specified number of inner layers without shearing or stretching. To illustrate this capability, a prescribed design change involving two triangular bumps was made to the axial distribution of radius for the SEEB body of revolution configuration. For this configuration, the core grid outer boundary was very close to the original body to improve signature propagation. Figure 18 shows the symmetry plane for the core grid for the baseline and design geometries, with the latter showing the protrusion of the core grid outer boundary required to smoothly absorb the changes from the modified surface. Figure 19 illustrates how these changes were then absorbed into a collar grid generated using the normal BG approach of starting the shearing and stretching at the first collar layer. The changes only extend to the fourth collar grid layer, so the negative cells created could be repaired, but a number of distorted cells were created in the process.

In an effort to avoid the distorted cells, a second collar grid was created from the same core grid but with ten layers of un-sheared cells that would contain all of the design changes. Figure 20 shows that this grid has eliminated the distorted cells, but it should be noted that with the use of the un-sheared inner layers the configuration is now farther away from the beginning of the sheared cells which may add dissipation to the signature. Also, as there are fewer stretched cells, the maximum stretching factor had to be increased to 1.4 to reach the same signature location with the same number of cells, a requirement for using solution restart. To evaluate the impact of using this approach, Euler flow solutions were obtained for the two grids at Mach $=1.6$ and an angle of attack of 0.0 degrees. Boom signatures were extracted 21.2 inches ( 1.2 body lengths) below the nose of the model and are compared in figure 21 . The baseline SEEB signature is also included to give perspective on the flow change resulting from the modifications. The results show that the signature from the normal BG approach generally has larger peaks than the one with the inner layers of un-sheared cells, particularly in the region just ahead of $x=40$ inches. This suggests that, even with some cell distortion, there appears to be less dissipation in the propagated signature for the original method, probably because of the earlier access to sheared cells. Additional test cases showed that the negative cells could still be eliminated using the original method even with the front bump height increased by a factor of 2.5 . As it is anticipated that most design changes for a real aircraft case would be considerably smaller than this, the recommendation for now would be to use the original method.

Another difference between boom and drag design using CDISC is that the target pressures are not on the surface but at a remote signature location. As mentioned earlier, the NFTARG code was created to try to relate the changes needed to match a boom target signature to geometry changes on the model. In order to test the ray-tracing approach 
used in NFTARG, a design case was set up using the baseline BG grid for the SEEB configuration discussed above. A target pressure distribution was defined by scaling the $\mathrm{x}$ coordinates of the baseline (no alpha increment) on-track computed boom signature for the LM1021 case shown in figure 17 to cover the shaped forebody portion of the SEEB model back to $\mathrm{x}=18.4$ inches. The pressure increments were converted to pressure coefficients for compatibility with CDISC and interpolated to every other grid point along the SEEB keel line. The interpolation caused some loss in signature detail, but the resulting target distribution should still provide an adequate check of the method. The case was run in Euler mode at a free-stream Mach number of 1.6 and zero degrees angle of attack.

The baseline, target and final design boom signatures are shown in figure 22. Eighty design cycles were needed as the design had to be under-relaxed in the early cycles to stabilize the process. The targets are matched surprisingly well, considering the multiple features in target signature from the different components (fuselage, wing, nacelle, etc.) of the LM1021, a fairly complete aircraft configuration. The ray tracing appears to have been successful, with the primary discrepancy being a small wave beginning near $\mathrm{x}=0.1$. This wave persisted from early cycles while the design in other regions moved fairly smoothly toward the target pressures. The reason for this discrepancy is still under investigation.

The resulting axial distribution of radius is compared with the baseline in figure 23 . The local geometry changes required to match the multiple peaks in the signature can be seen. As the CDISC approach is applied directly to every other grid point along the fuselage keel line, this level of resolution was not a problem. Methods that rely on shape functions to change the geometry may have a harder time achieving this level of agreement. As noted by $\mathrm{Li}^{20}$, attempting optimization in high-resolution design space usually requires significant smoothing to be added. Finally, it should be noted that the design changes for this case were either small enough or occurred in regions away from the core grid outer boundary so that the standard BG approach (i.e., begin stretching in the first collar grid layer) could be used with no resulting cell distortion. It should be noted that, although in the above example the design changes kept the body axi-symmetric, this is not a requirement.

Two interesting questions arise from the above design result that time constraints did not allow to be investigated. The first is how does the geometry from the design compare with one derived from an equivalent area method ${ }^{21}$ for the LM1021? The second question is could the fuselage of the LM1021 be redesigned to match the boom signature for the SEEB (i.e., do the reverse design problem)? The second question in particular addresses the use of wave cancellation for boom reduction and is currently being explored. If the approach shows promise for on-track signature improvement, the effects on off-track as well as off-design conditions will need to be considered.

\section{Concluding Remarks}

The parametric studies conducted for the COB and BG codes in general confirm that the overall process produces grids that give accurate sonic boom signature predictions. While the current study used the two methods in sequence, either code could be paired with a different method if desired (e.g., BG can be used to create a collar grid about a core grid generated by an method other than COB/VGRID). Currently, the one restriction is that the grids be defined using the VGRID format. In addition, the results relative to grid spacing should be applicable to any methods used in a similar boom grid generation process. Summary comments and recommendations on each of the codes are as follows:

The COB method provided a more robust approach to generating the core grid than the previous method, allowing cases that could not achieve the desired radial distance for the collar grid in BG to be able to do so. Based on the parametric studies, it is recommended that the automated method for placing the core grid outer boundary close to the bottom of the fuselage be used. It appears that an axial spacing under the fuselage of the body length divided by 300 provides good signature resolution with as small a grid as possible. The 3-zone approach to axial spacing is probably a good starting point, with the multi-zone capability as a useful option to provide further resolution of key areas, if needed, with minimal additional grid cells. The rectangular outer boundary (ROB) option in COB appears very promising, providing significantly smaller grids than with the original cylindrical outer boundary without loss of accuracy, at least for the on-track cases examined. The new option in COB for extending dense grid to a given off-track angle increased the grid size without providing any significant improvement in off-track signatures and is not recommended at this time for cylindrical grids, though it is still being assessed for rectangular outer boundaries. 
For the BG code, it appears that the radial distance to the collar grid outer boundary can be set to the desired signature location for typical cases in propagation studies, with no cushion needed to avoid reflection issues. For wind tunnel test conditions where smaller distances on the order of one body length are encountered, this finding may not hold true. It is strongly recommend that the alpha option in BG be used, even for very small angles. The maximum stretching factor of 1.0 recommended in the previous study was confirmed as giving the best combination of accuracy and efficiency. The off-track grid focusing option did not provide any noticeable improvement in offtrack signature accuracy for the cases shown, but as it doesn't involve any increase in grid size and did show some benefit in the previous study, it is suggested that it be used. It may especially prove useful with core grids from the ROB approach, where the span-wise growth of grid spacing is not required to be as small as with the COB method.

Finally, in regard to design, it appears that the option in BG for un-stretched inner collar grid layers does not provide a benefit and may actually increase signal dissipation. The results from the SEEB design case with CDISC indicate that the ray-tracing approach used in the NFTARG code provides an effective sonic boom design capability, with CDISC giving a close match to the complex target pressure distribution. Further evaluations of this approach are underway at this time.

\section{Acknowledgments}

The work done was sponsored by NASA Fundamental Aeronautics Program Supersonics Project, including funding under contract NNL09AM01T.

\section{References}

${ }^{1}$ http://www.aeronautics.nasa.gov/fap/2012-PRESENTATIONS/SUP_2012_508.pdf

${ }^{2}$ Graham, D., Dahlin, J., Meredith, K., and Vadnais, J.: Aerodynamic Design of Shaped Sonic Boom Demonstration Aircraft, AIAA 2005-0008, January 2005.

${ }^{3}$ Howe, D. C., Waithe, K. A., and Haering, E. A.: Quiet SpikeTM Near-Field Flight Test Pressure Measurements with Computational Fluid Dynamics Comparisons, AIAA 2008-0128, January 2008.

${ }^{4}$ Magee, Todd E.; Shaw, Stephen G.; and Fugal, Spencer R.: Experimental Validations of a Low-Boom Aircraft Design, AIAA 2013-0646, January 2013.

${ }^{5}$ Morgenstern, John M.; Buonanno, Michael; and Marconi, Frank, PhD: Full Configuration Low Boom Model and Grids for a 2014 Sonic Boom Prediction Workshop, AIAA 2013-0647, January 2013.

${ }^{6}$ Magee, Todd E.; Fink, Lawrence E.; Fugal, Spencer R.; Adamson, Eric E.; and Shaw, Stephen G.: Boeing N+2 Supersonic Experimental Validation Phase II Program, AIAA 2014-2137, June 2014.

${ }^{7}$ Morgenstern, John M.; Buonaano, Michael; Chai, Sunny; and Marconi, Frank: Overview of Sonic Boom Reduction Efforts on the Lockheed Martin N+2 Supersonic Validations Program, AIAA 2014-2138, June 2014.

${ }^{8}$ Cliff, Susan E.; Durston, Donald A.; Elmiligui, Alaa A.; Jensen, James C.; and Chan, William M.: Computational and Experimental Assessment of Models for the First AIAA Sonic Boom Prediction Workshop, AIAA 2014-0560, January 2014.

${ }^{9}$ Park, Michael A.; and Morgenstern, John M.: Summary and Statistical Analysis of the First AIAA Sonic Boom Prediction Workshop, AIAA 2014-2006, June 2014.

${ }^{10}$ Park, Michael A.; Campbell, Richard L.; Elmiligui, Alaa; Cliff, Susan E.; and Nayani, Sudheer N.: Specialized CFD Grid Generation Methods for Near-Field Sonic Boom Prediction, AIAA 2014-0115, January 2014.

${ }^{11}$ Frink,N. T.; Pirzadeh, S. ${ }^{11}$ Frink, N.T., Pirzadeh, S.Z., Parikh, P.C., Pandya, M.J., and Bhat, M.K.: "The NASA Tetrahedral Unstructured Software System”, The Aeronautical Journal, Vol. 104, No. 1040, October 2000, pp.491-499.

${ }^{12} \mathrm{Li}$, Wu; Campbell, Richard; Geiselhart, Karl; Shields, Elwood; Nayani, Sudheer; and Shenoy, Rajiv: Integration of Engine, Plume, and CFD Analyses in Conceptual Design of Low-Boom Supersonic Aircraft, AIAA 2009-1171, January 2009.

${ }^{13}$ Nayani, Sudheer N.; and Campbell, Richard L.: Evaluation of Grid Modification Methods for On- and Off-Track Sonic Boom Analysis, AIAA 2013-0798, January 2013.

${ }^{14}$ Ordaz, Irian; Li, Wu; and Campbell, Richard L.: Automated Tetrahedral Mesh Generation for CFD Analysis of Aircraft in Conceptual Design, AIAA 2014-0118, January 2014.

${ }^{15}$ Roe, P., "Characteristic Based Schemes for the Euler Equations," Annual Review of Fluid Mechanics, Vol. 18, 1986, pp. $337-$ 365 .

${ }^{16}$ Menter, F.R., "Improved Two-Equation k-omega Turbulence Models for Aerodynamic Flows,” NASA TM-103975, October 1992.

${ }^{17}$ Campbell, Richard L., "Efficient Viscous Design of Realistic Aircraft Configurations,” AIAA-98-2539, June 1998. 
${ }^{18}$ Campbell, Richard L.; Campbell, Matthew L.; and Streit, Thomas: "Progress Toward Efficient Laminar Flow Analysis and Design.” AIAA-2011-3527, June 2011.

${ }^{19}$ Aftosmis, M. J., Berger, M. J., and Adomavicius, G.,: A Parallel Multilevel Method for Adaptively Refined Cartesian Grids with Embedded Boundaries, AIAA 2000-808, January 2000.

${ }^{20} \mathrm{Li}, \mathrm{W}$.; Krist, S.; and Campbell, R.: Transonic Airfoil Shape Optimization in Preliminary Design Environment, AIAA 20044629, August 2004.

${ }^{21} \mathrm{Li}, \mathrm{Wu}$; and Rallabhandi, Sriram: Inverse Design of Low-Boom Supersonic Concepts Using Reversed Equivalent-Area Targets, AIAA 2011-3498, June 2011 


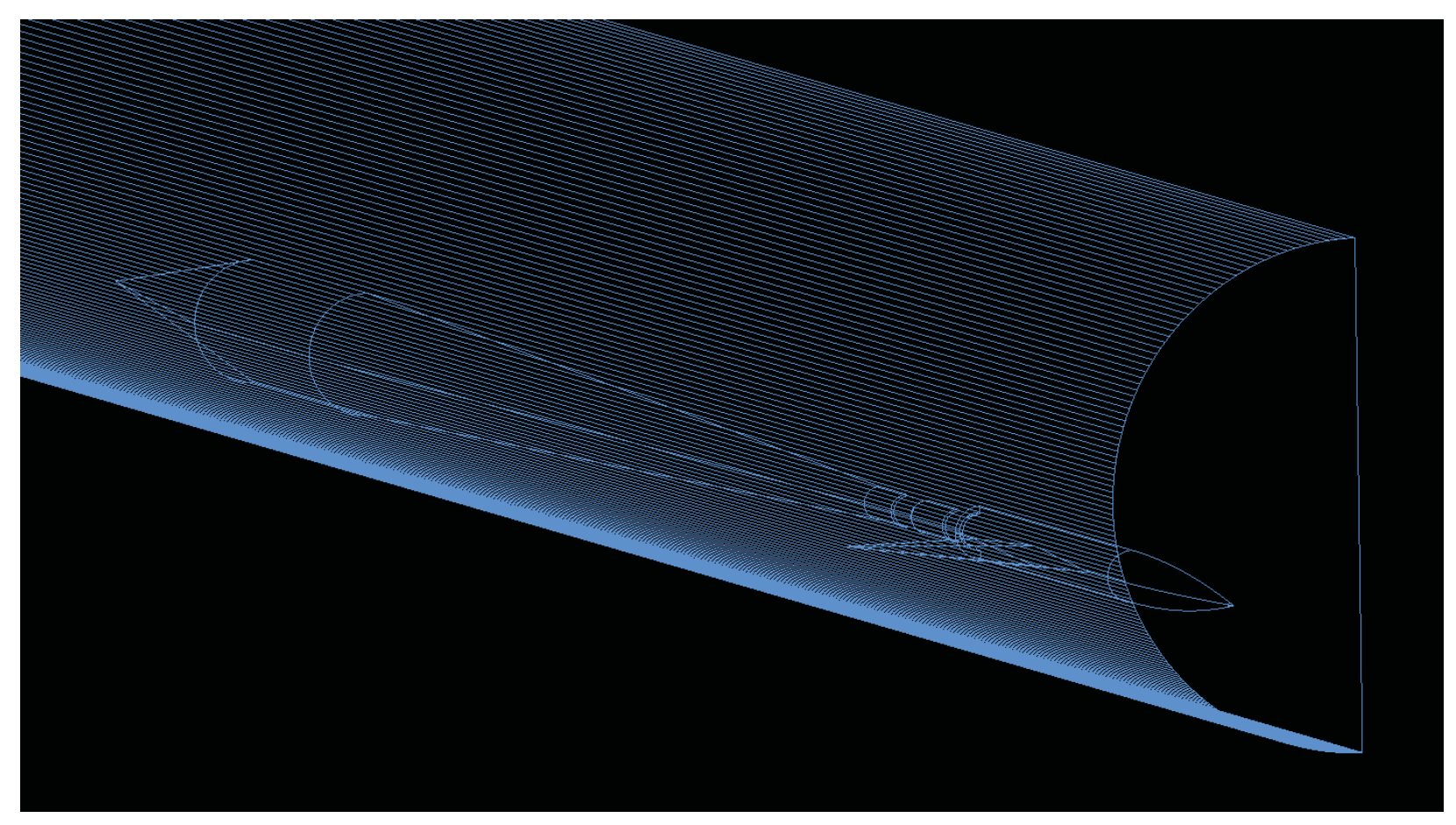

a) Core grid cylindrical outer boundary patches

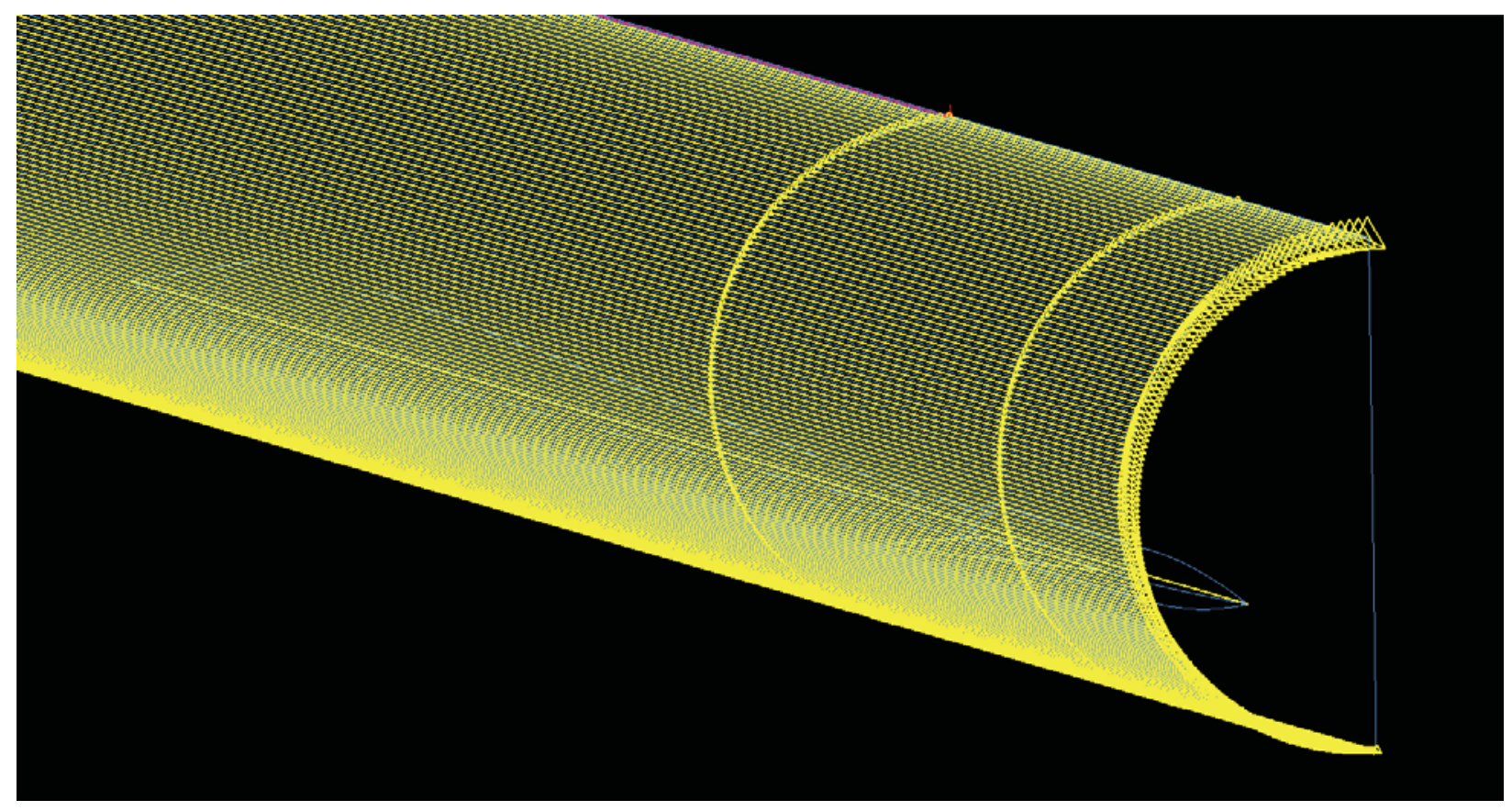

b) Outer boundary line sources (triangles denote local grid size)

Figure 1. COB output for a delta wing body configuration.

American Institute of Aeronautics and Astronautics 


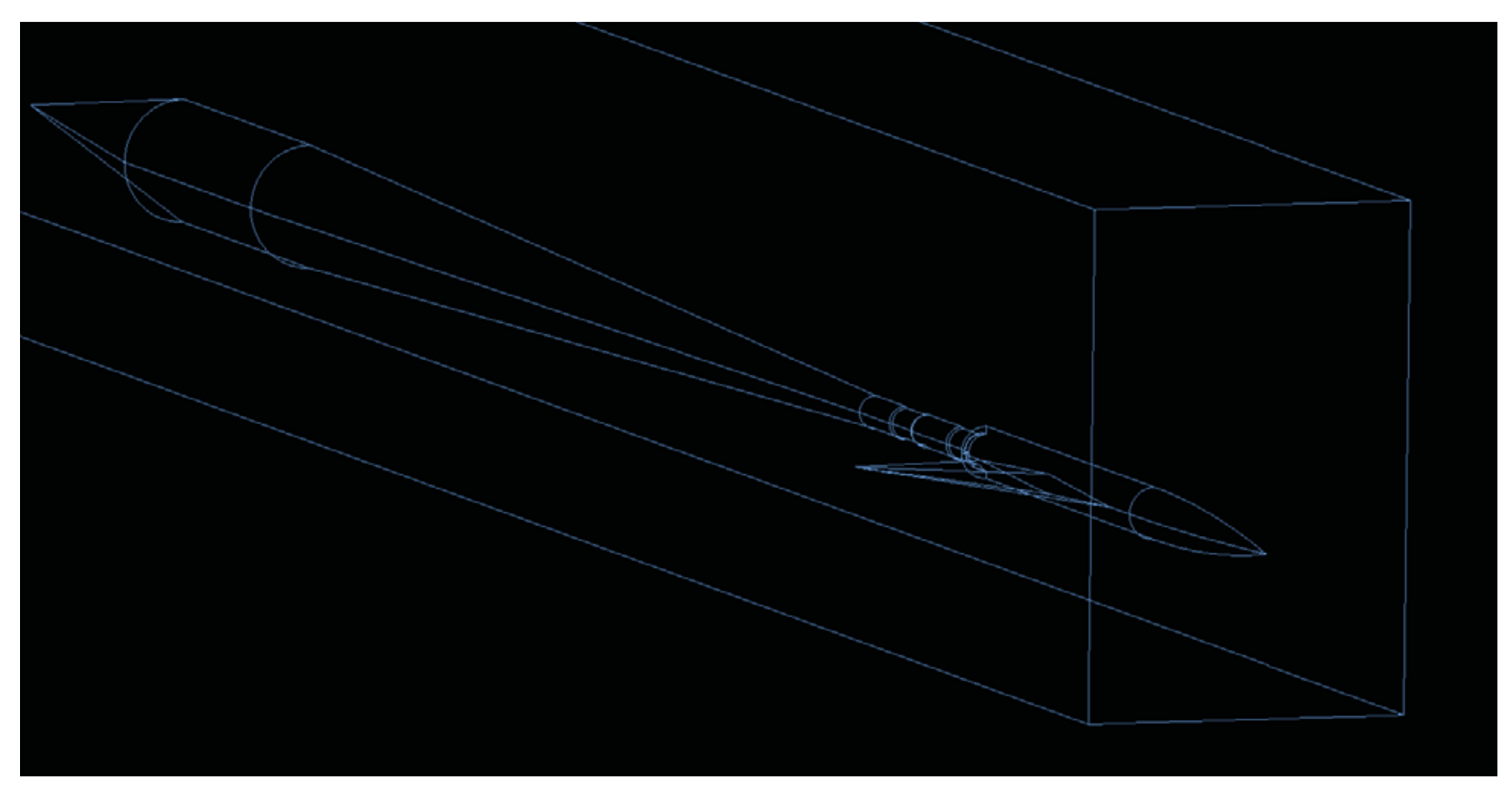

a) Core grid rectangular outer boundary patches

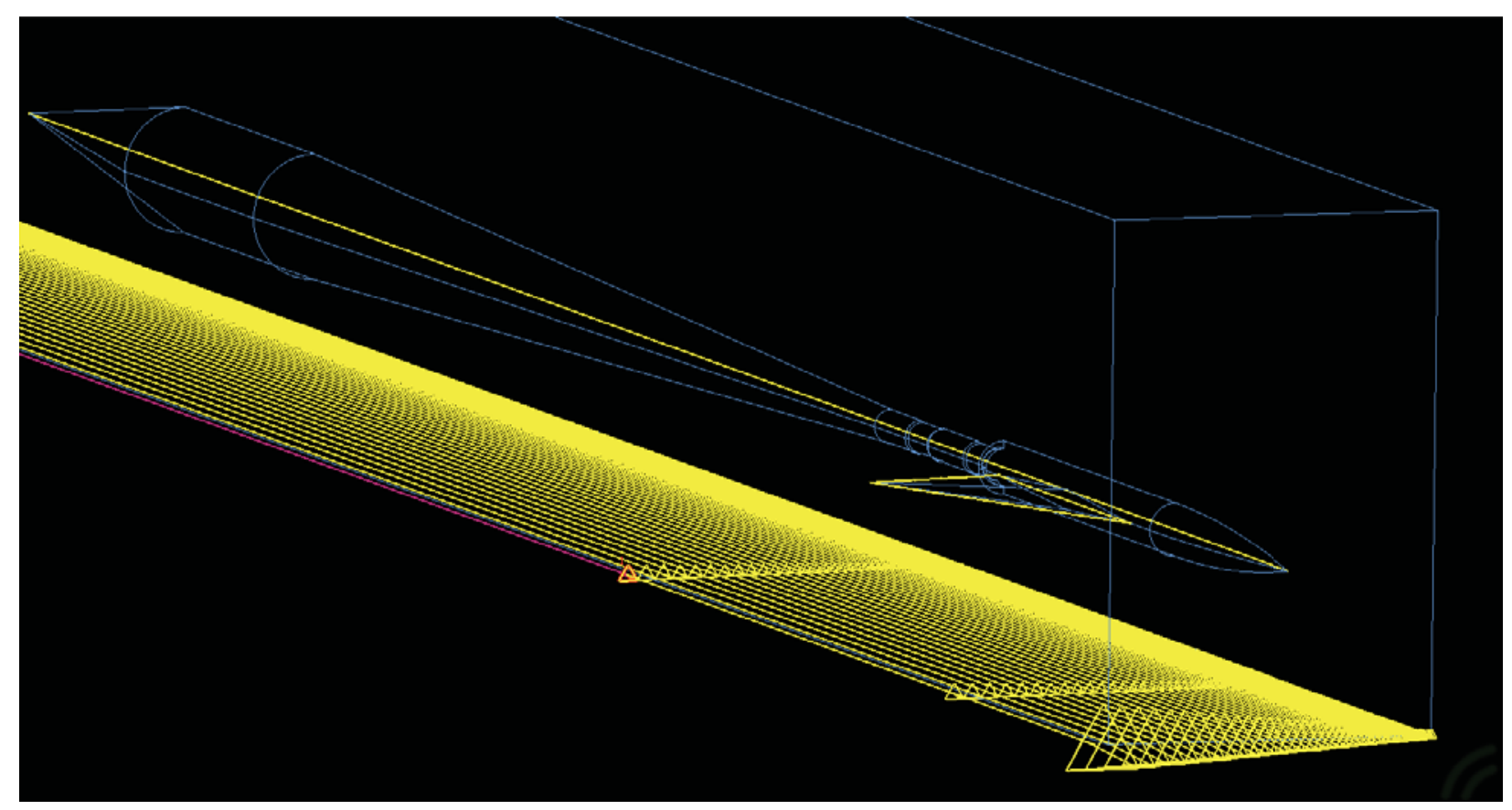

b) Outer boundary line sources (triangles denote local grid size)

Figure 2. Patches and sources created using ROB option for a delta wing body configuration. 


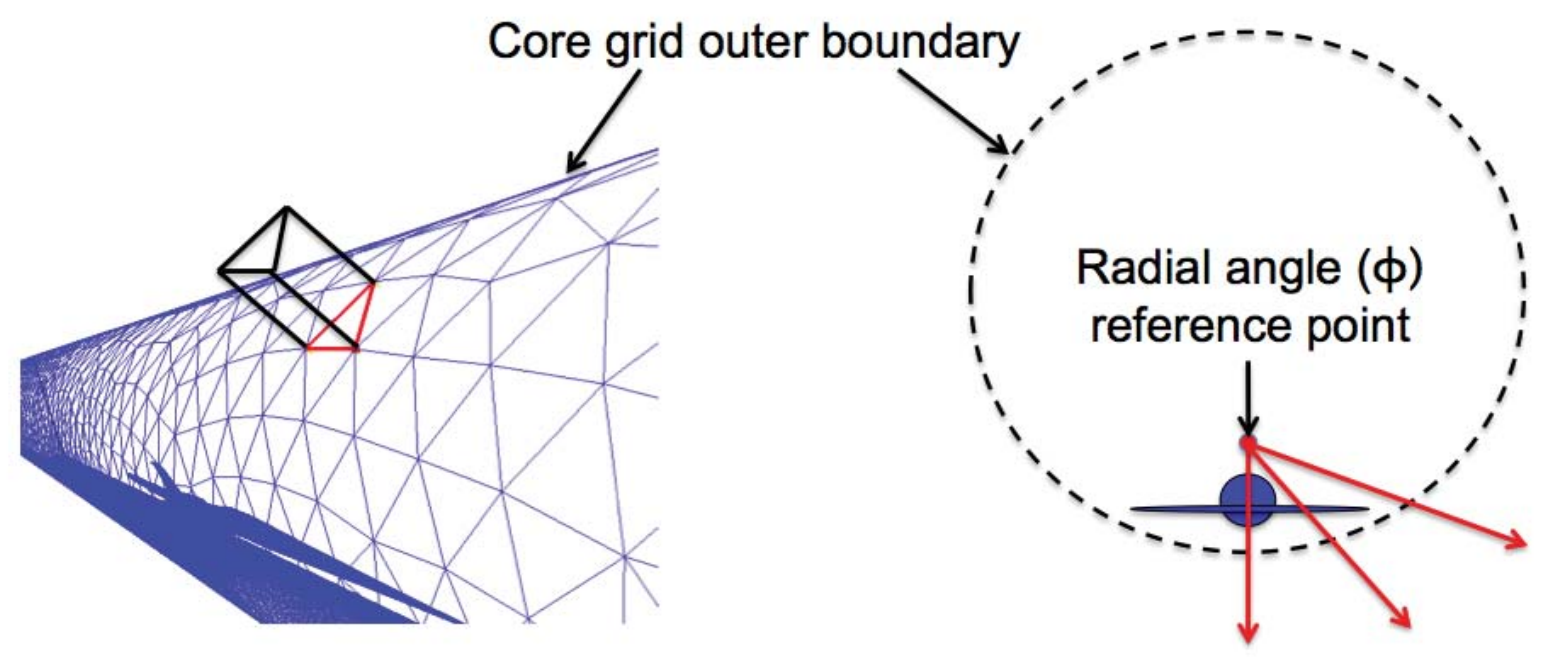

Figure 3. Prism extrusion process in the BG grid methodology.

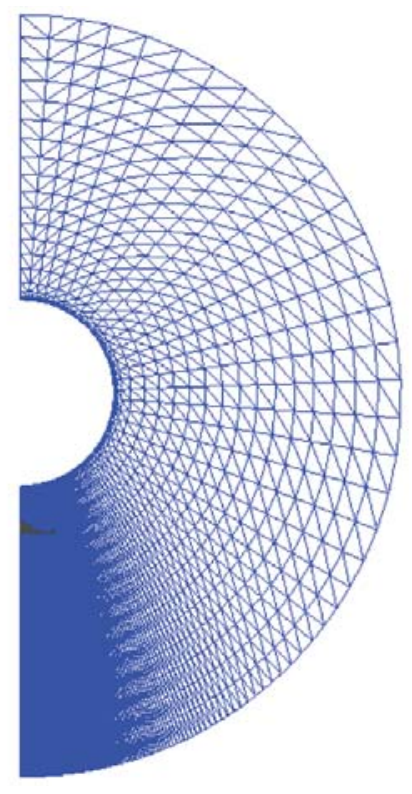

a) On-track

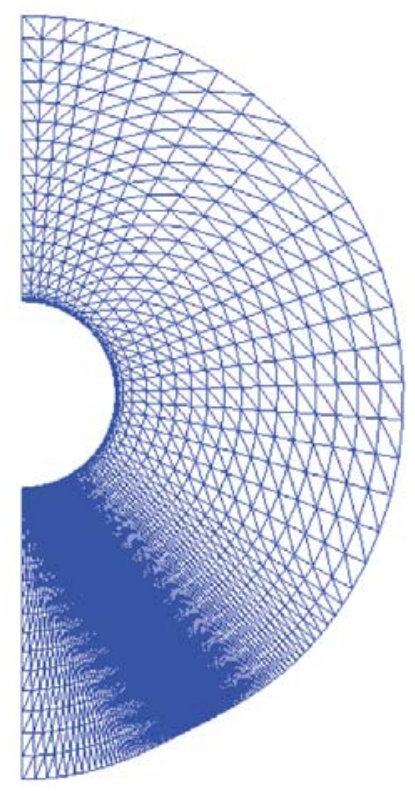

b) Off-track

Figure 4. Effect of BG grid-focusing option for off-track analysis on collar grid inflow plane. 


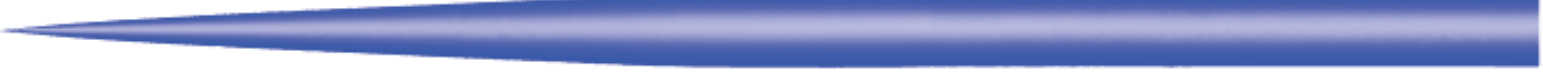

a) SEEB

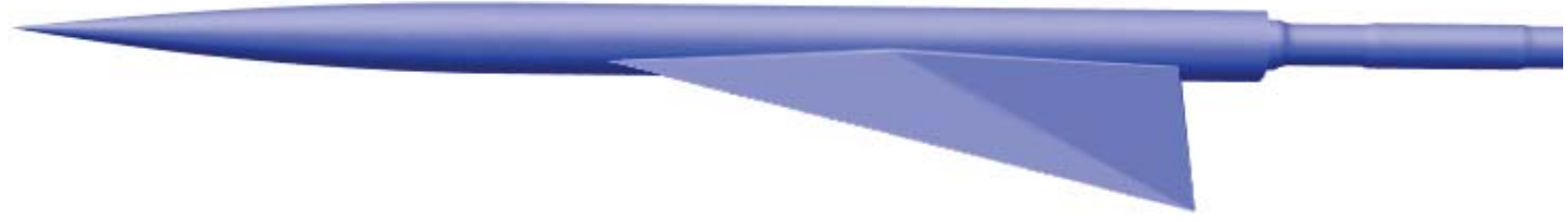

b) DWB

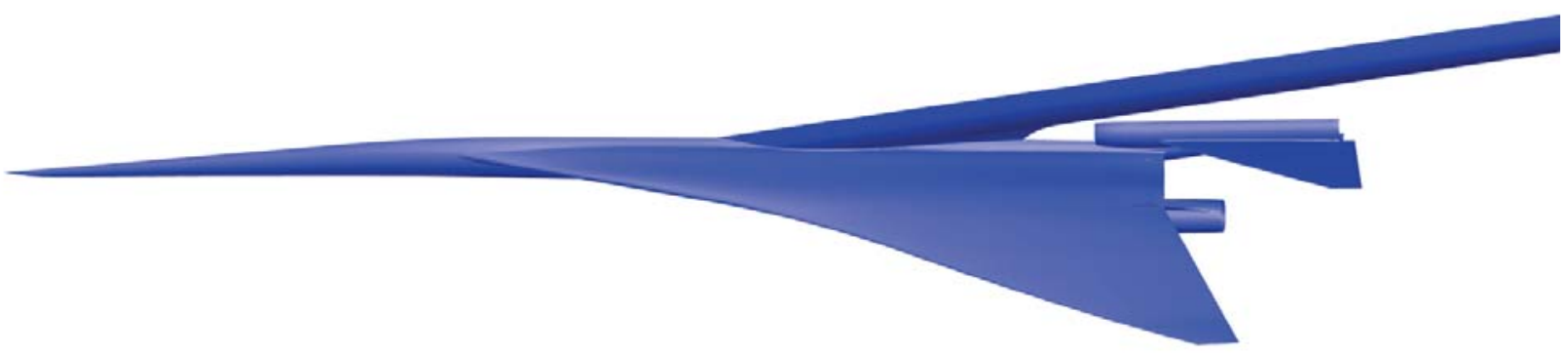

c) LM1021

Figure 5. CFD Models used in evaluations. 


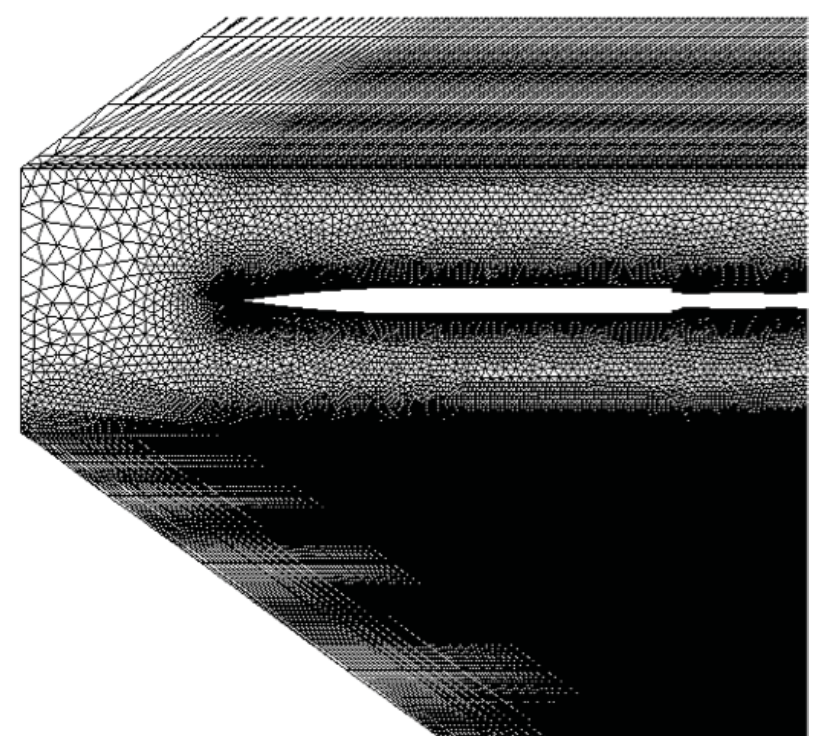

a) Model centered in core grid

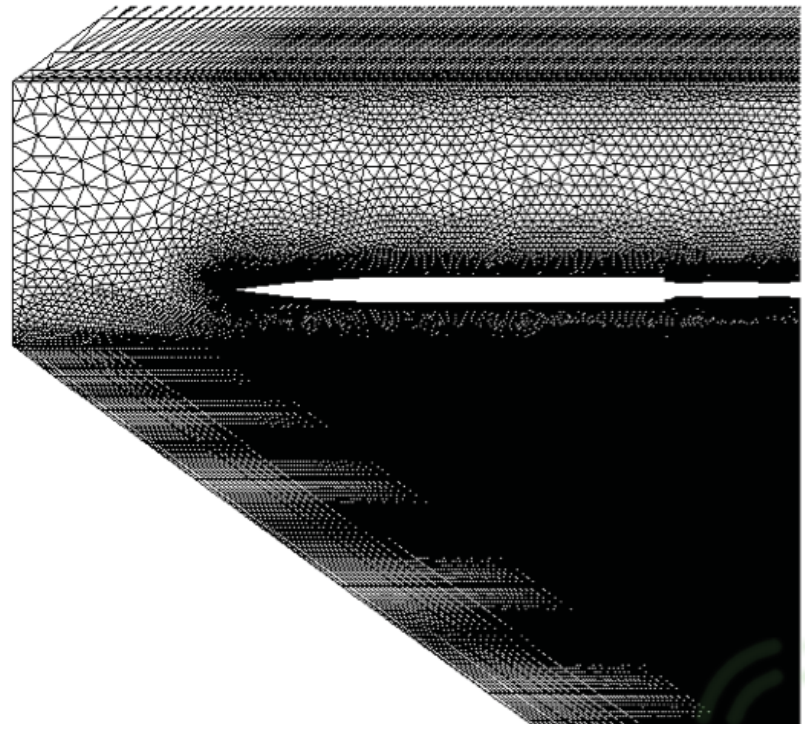

b) Automated placement of model by $\mathrm{COB}$

Figure 6. Symmetry plane grids for model vertical location study.

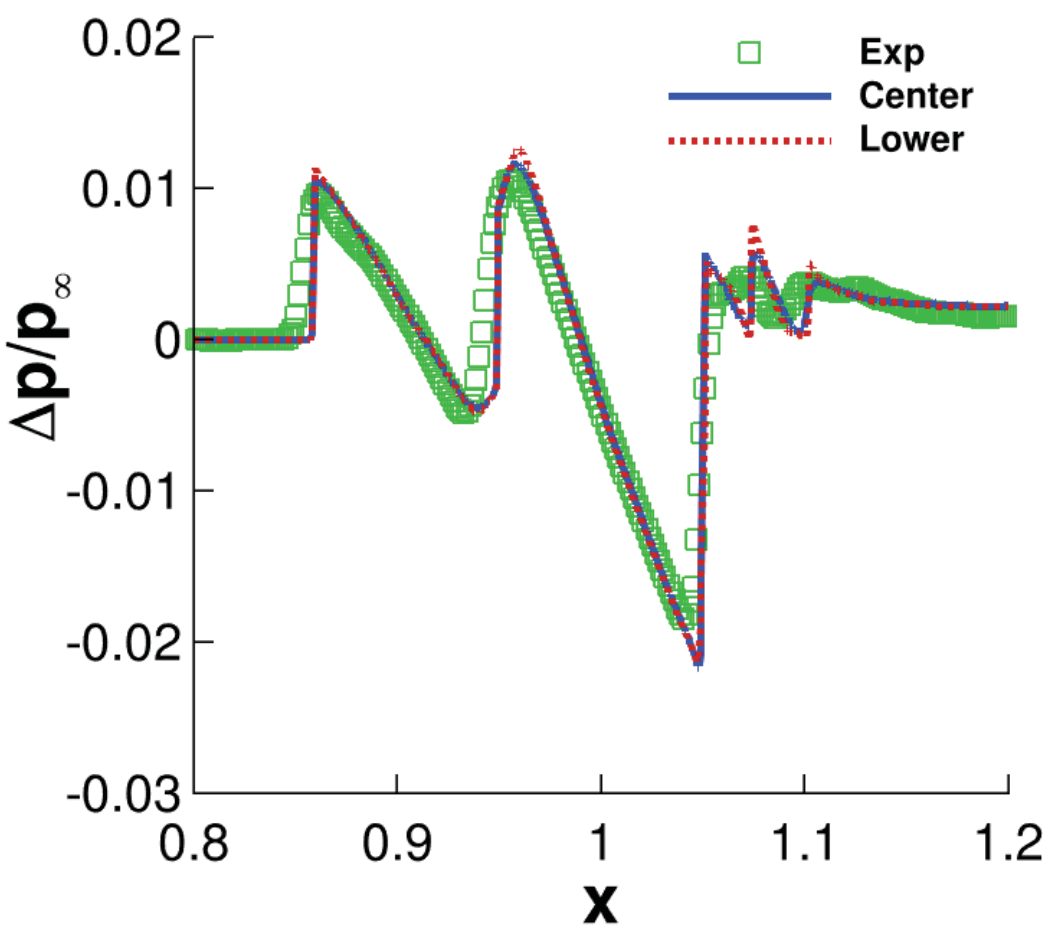

Figure 7. Effect of model vertical location on on-track signature at $\mathrm{H} / \mathrm{L}=3.6$ below the nose of the DWB configuration. ( $x$ is in meters, origin at fuselage nose). 

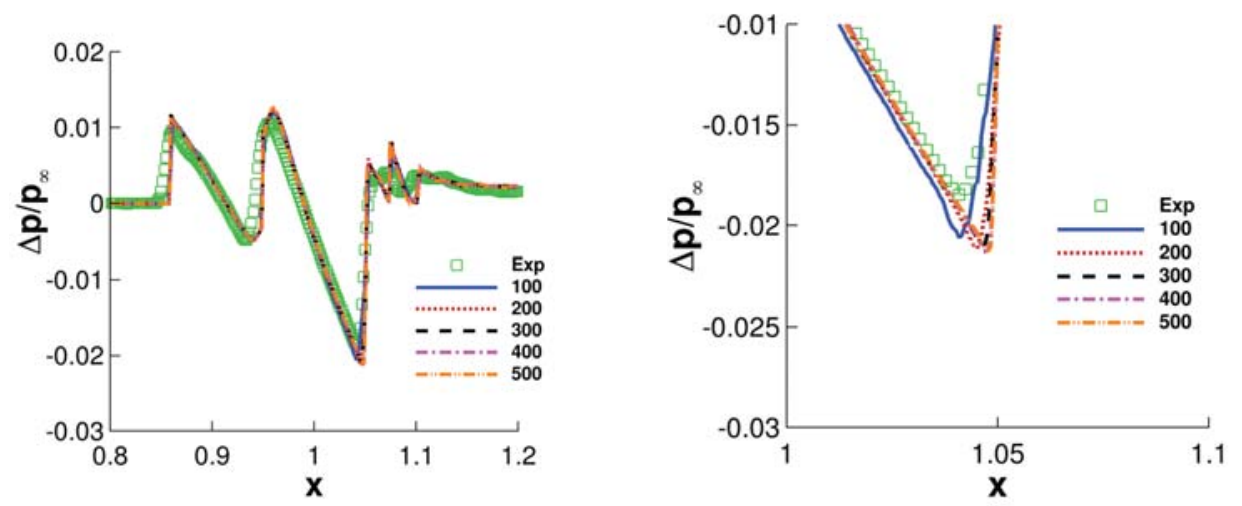

a) On-track
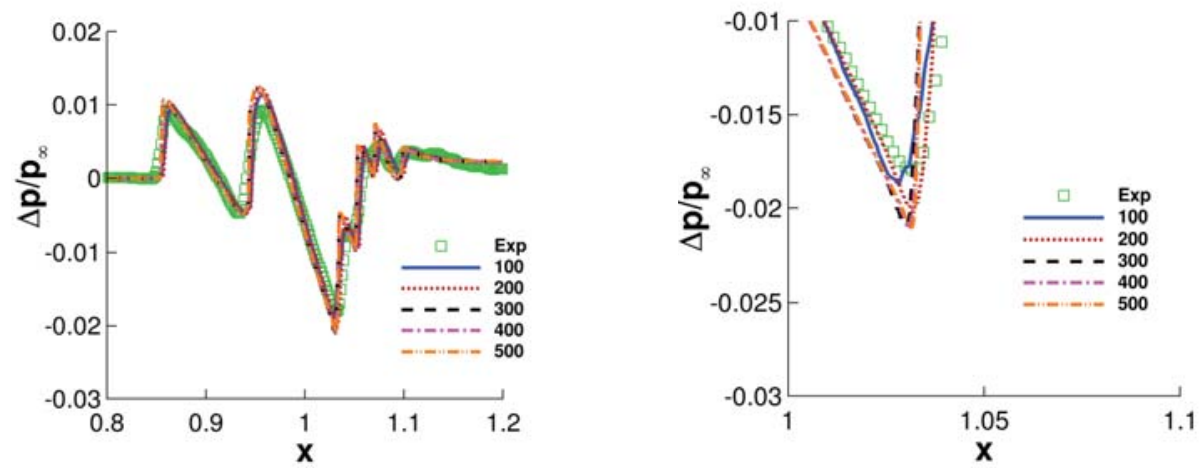

b) Off-track angle $=30$ degrees
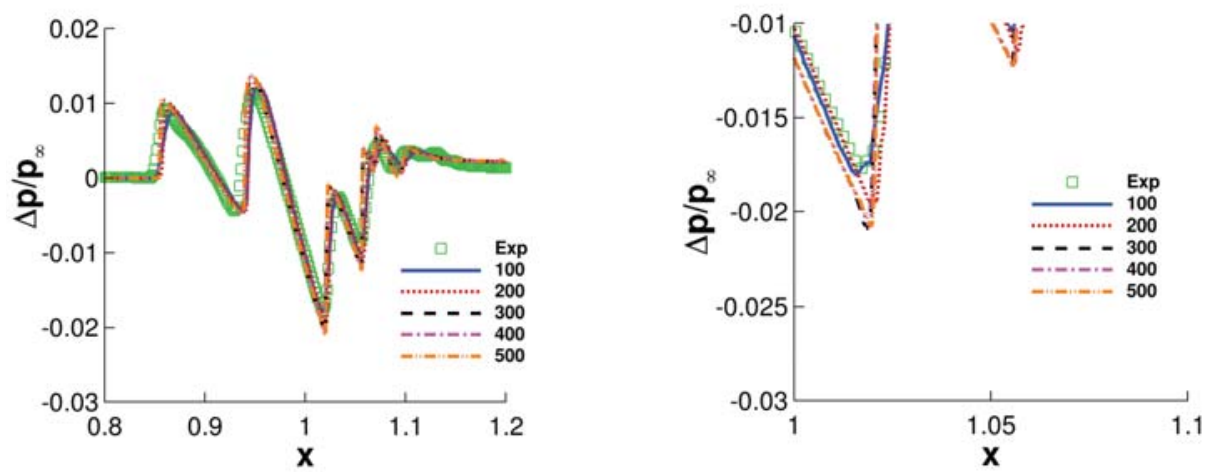

c) Off-track angle $=60$ degrees

Figure 8. Effect of axial spacing parameter on on- and off-track signatures at $H / L=3.6$ below the nose of the DWB configuration. ( $x$ is in meters, origin at fuselage nose). 


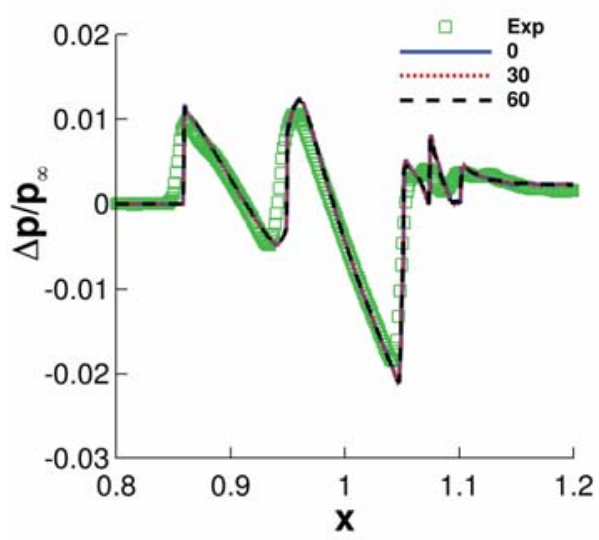

a) No extended spacing

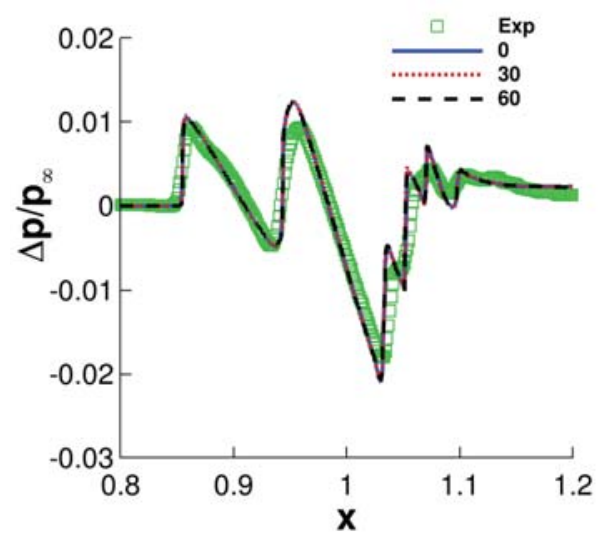

b) Spacing extended to 30 degrees

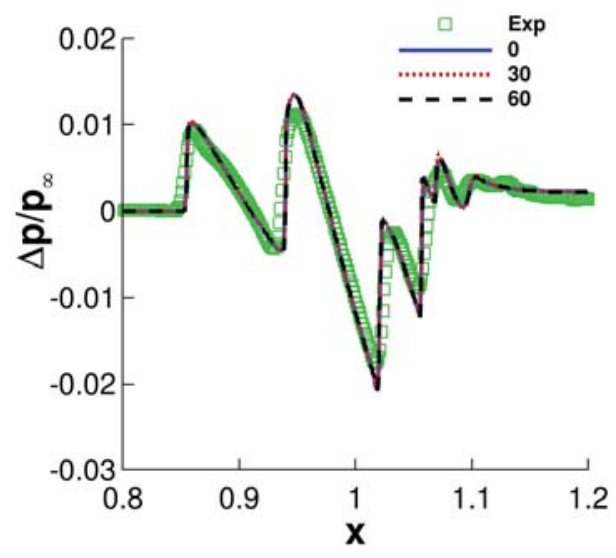

c) Spacing extended to 60 degrees

Figure 9. Effect of extended circumferential fine grid spacing in COB with corresponding BG focus angle on on- and off-track signatures at $\mathrm{H} / \mathrm{L}=3.6$ below the nose of the DWB configuration. ( $x$ is in meters, origin at fuselage nose). 


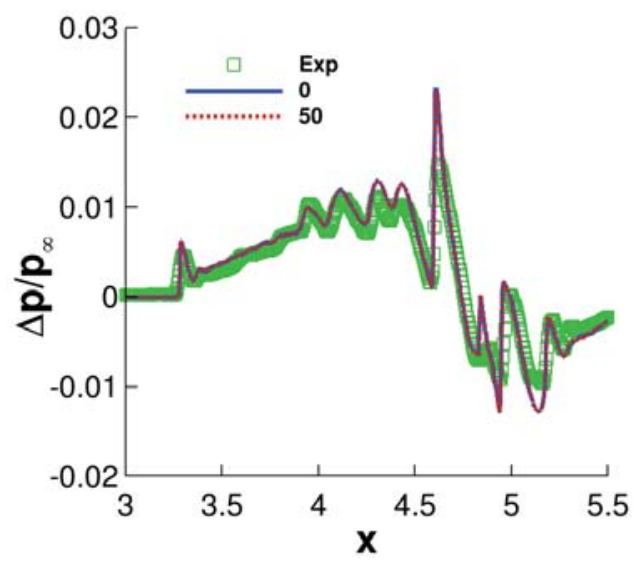

a) On-track

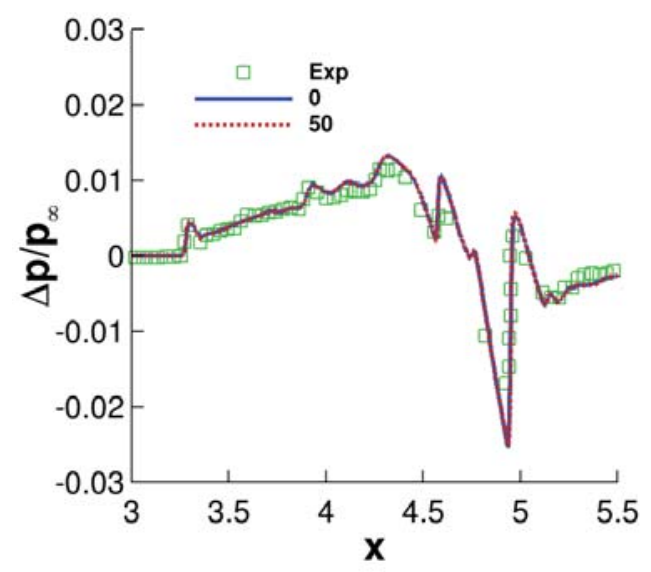

b) Off-track angle $=30$ degrees

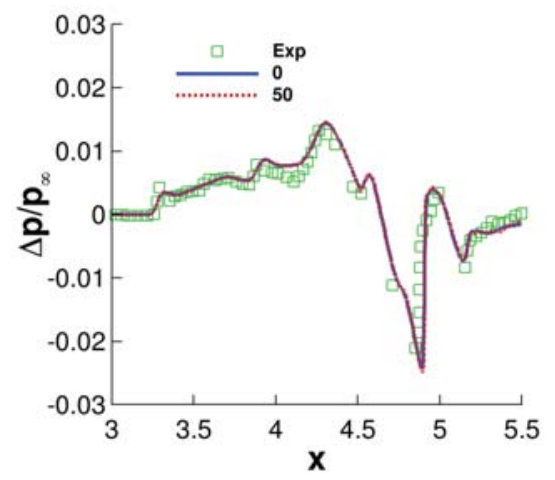

c) Off-track angle $=\mathbf{5 0}$ degrees

Figure 10. Effect of extended circumferential fine grid spacing in $\mathrm{COB}$ with corresponding BG focus angle on on- and off-track signatures at $H / L=1.4$ below the nose of the LM1021 configuration. ( $x$ is in meters, origin at fuselage nose). 


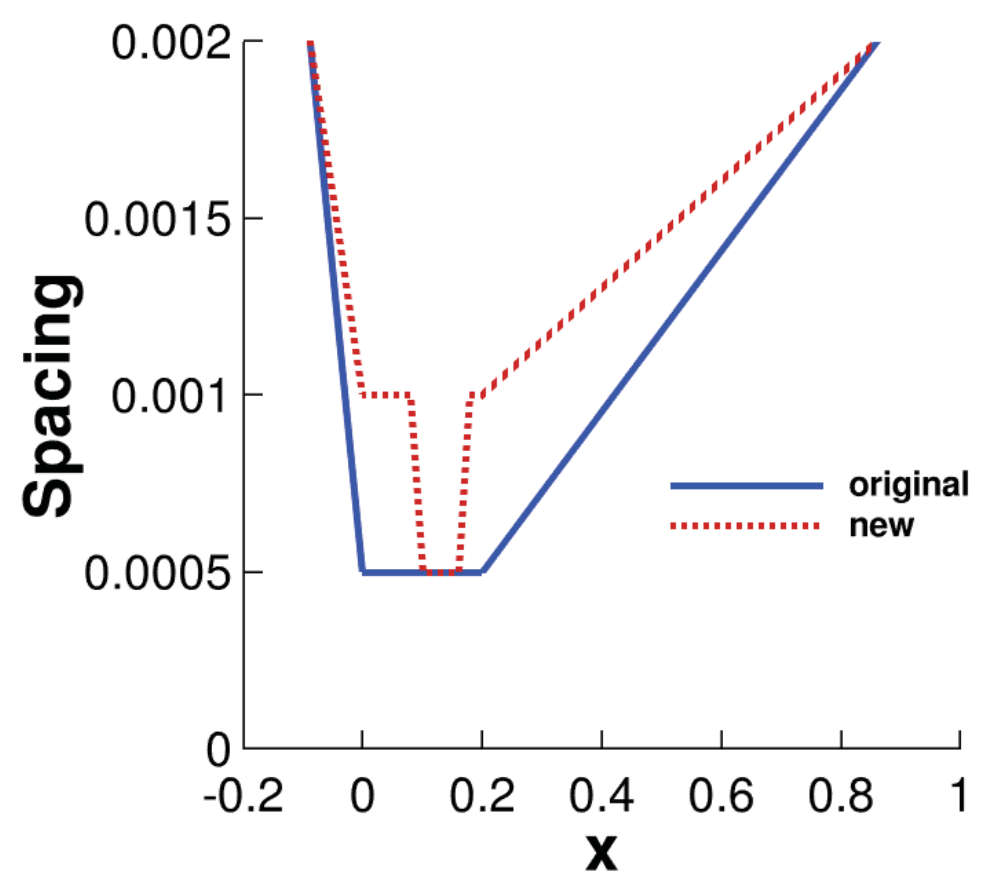

Figure 11. Axial distribution of grid spacing (in meters) for original and new multi-zone option in COB for the DWB configuration ( $x$ is in meters, origin at fuselage nose).

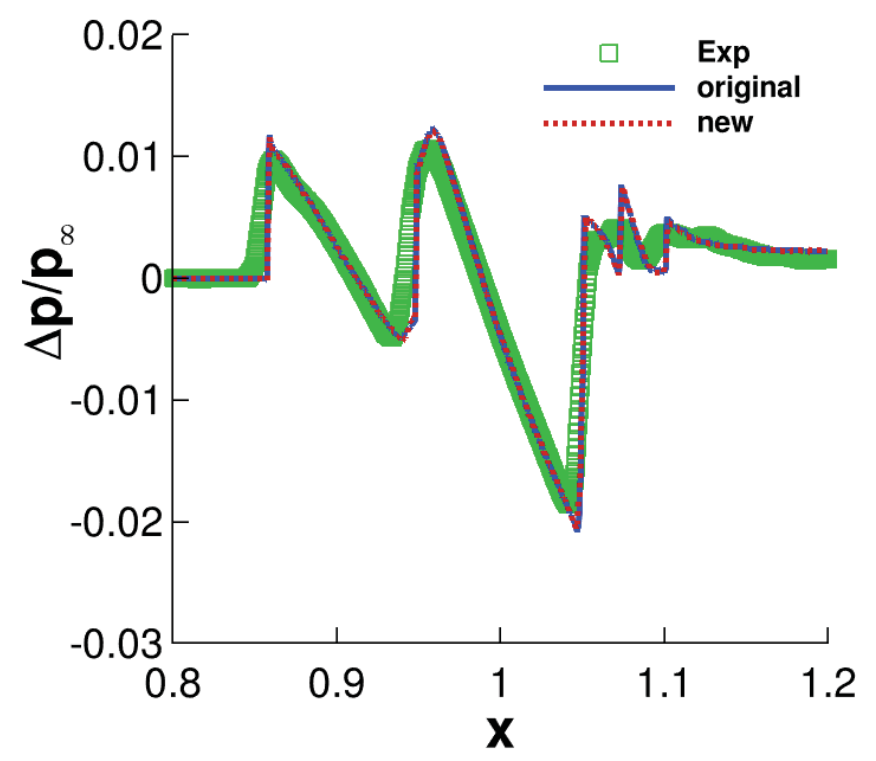

Figure 12. Effect of new multi-zone axial grid spacing in COB on on-track signatures at $\mathrm{H} / \mathrm{L}=3.6$ below the nose of the DWB configuration. ( $x$ is in meters, origin at fuselage nose). 


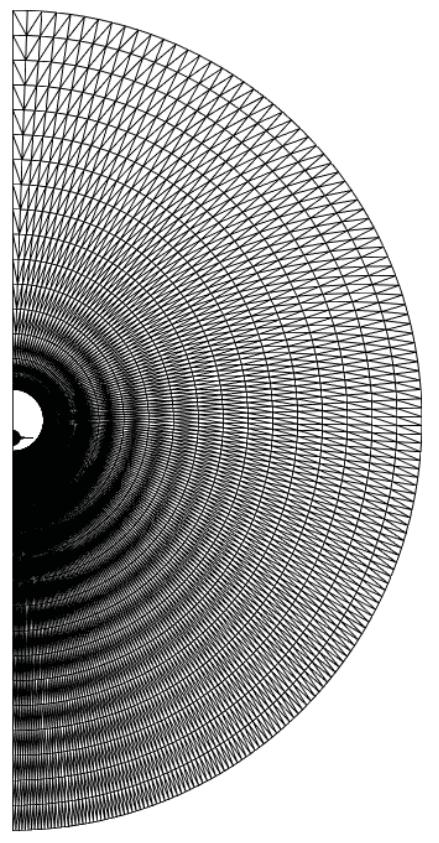

a) $\mathrm{COB}$

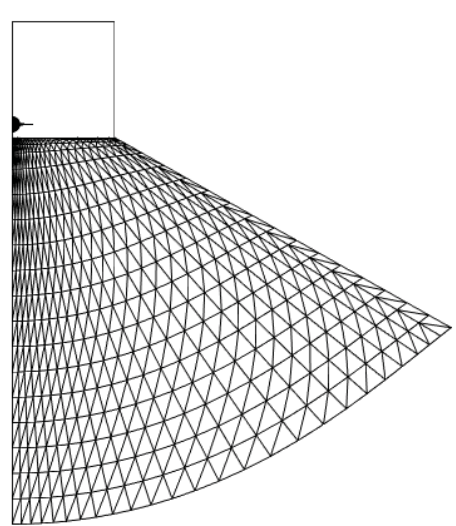

b) ROB

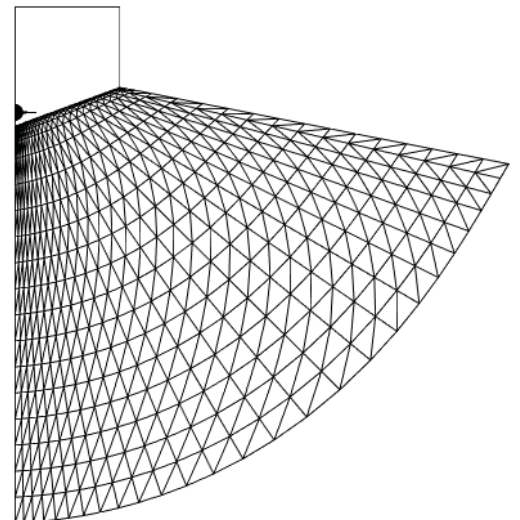

c) ROBT

Figure 13. Collar grid inflow planes generated using the COB, ROB and ROBT methods for the DWB configuration.

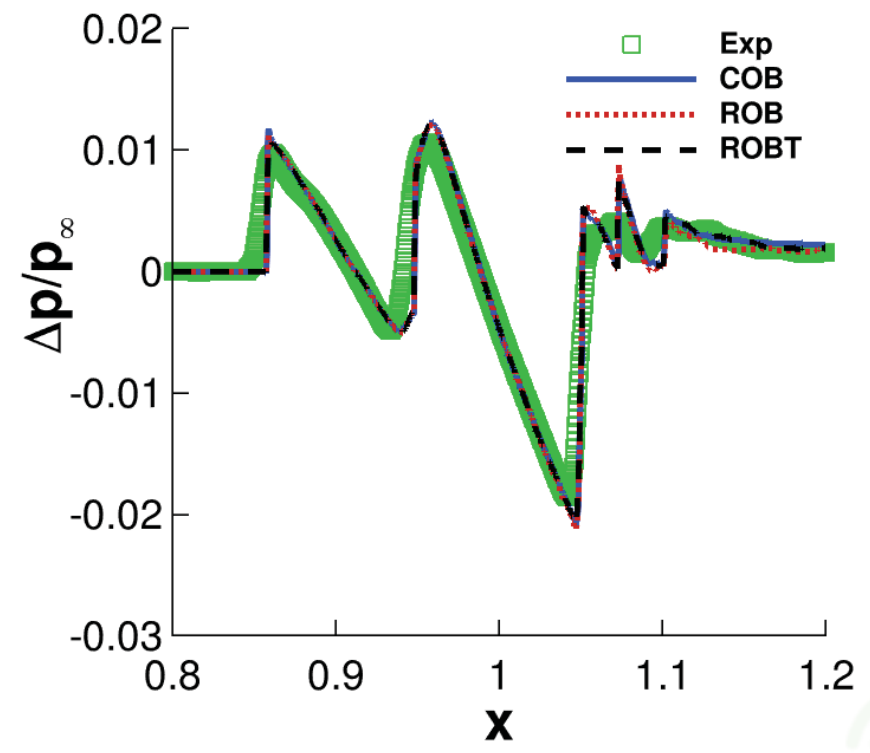

Figure 14. Effect of core grid outer boundary geometry on on-track signature at $H / L=3.6$ below the nose of the DWB configuration. ( $x$ is in meters, origin at fuselage nose). 


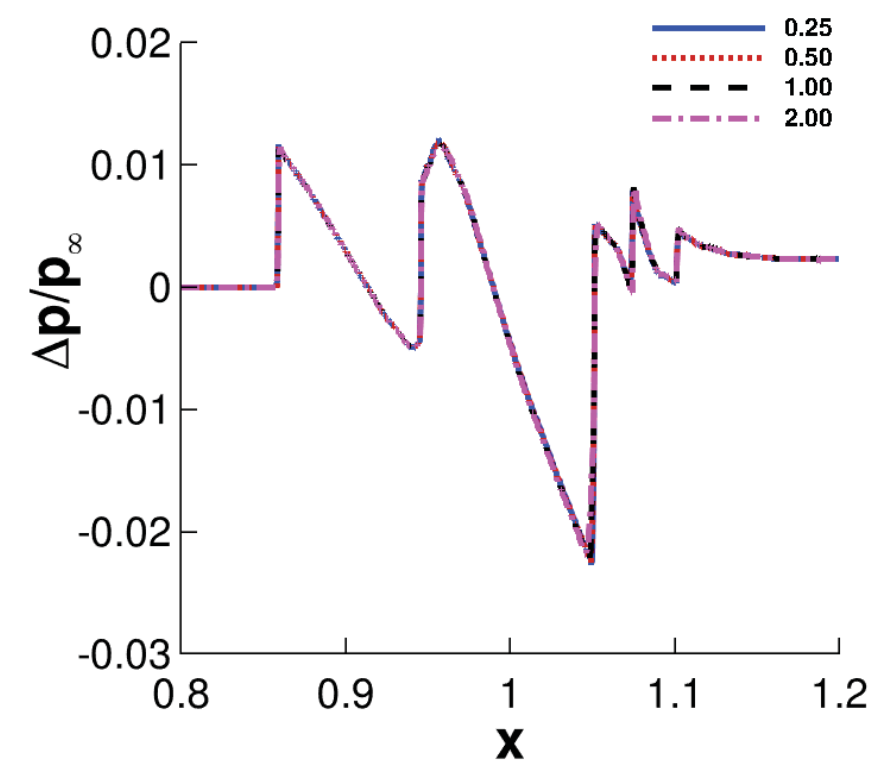

a) Full signature

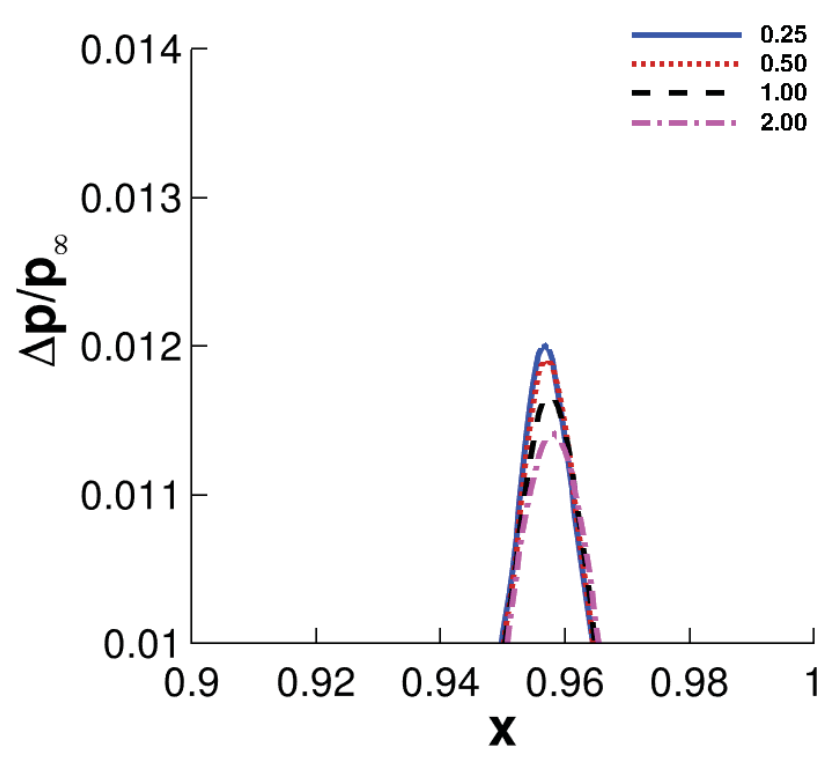

b) Close-up of peak

Figure 15. Effect of maximum stretching ratio in $B G$ on on-track signature at $H / L=3.6$ below the nose of the DWB configuration. ( $x$ is in meters, origin at fuselage nose). 


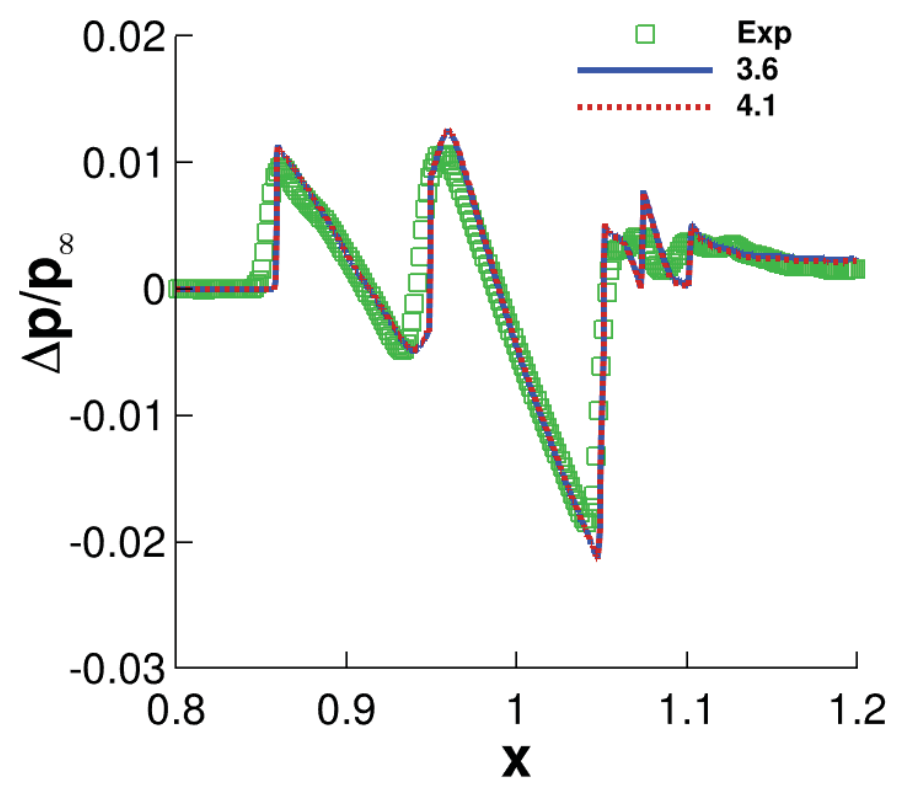

Figure 16. Effect of radial distance to the outer boundary (in body lengths) in BG on on-track signature at $\mathrm{H} / \mathrm{L}=3.6$ below the nose of the DWB configuration. ( $\mathrm{x}$ is in meters, origin at fuselage nose)

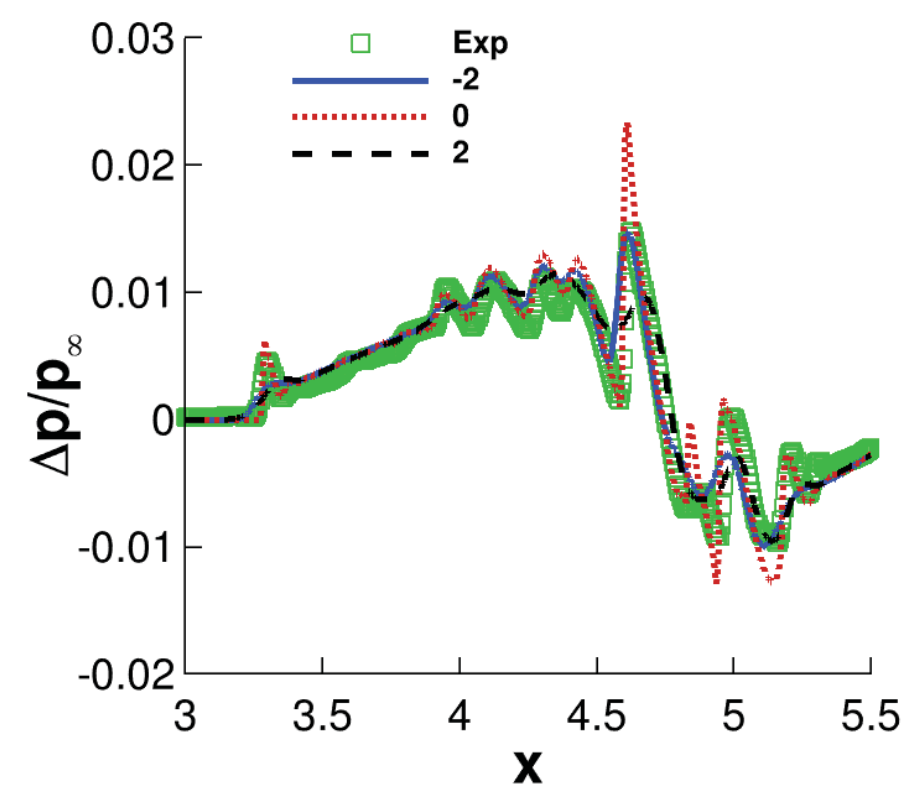

Figure 17. Effect of angle-of-attack parameter in $B G$ on on-track signature at $H / L=1.4$ below the nose of the LM1021 configuration. ( $x$ is in feet, origin at fuselage nose). 


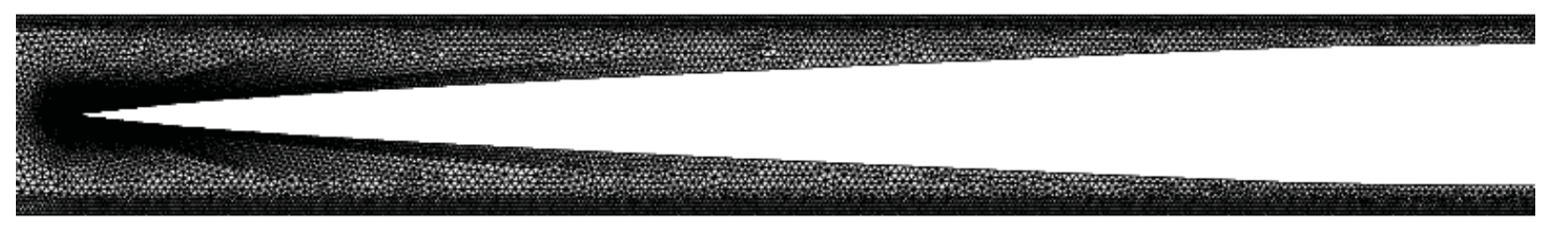

a) Baseline

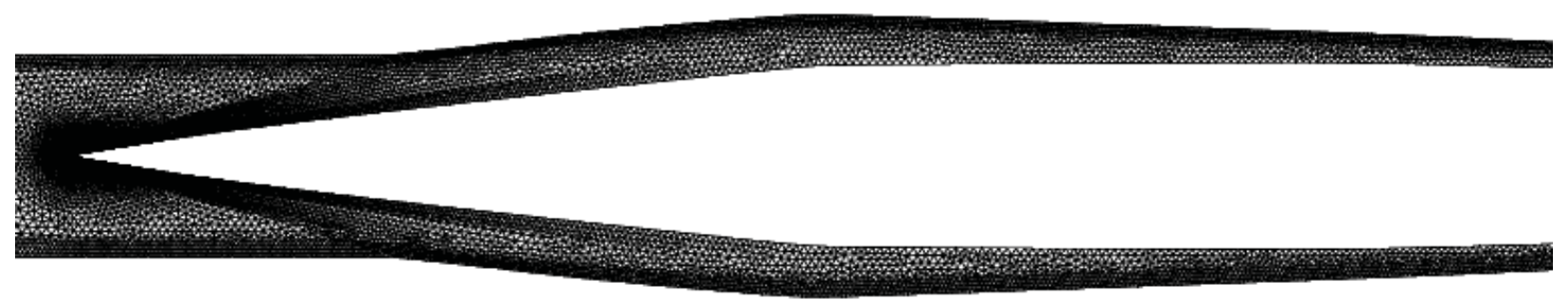

b) Modified

Figure 18. Core grid symmetry plane grid for SEEB modification case.

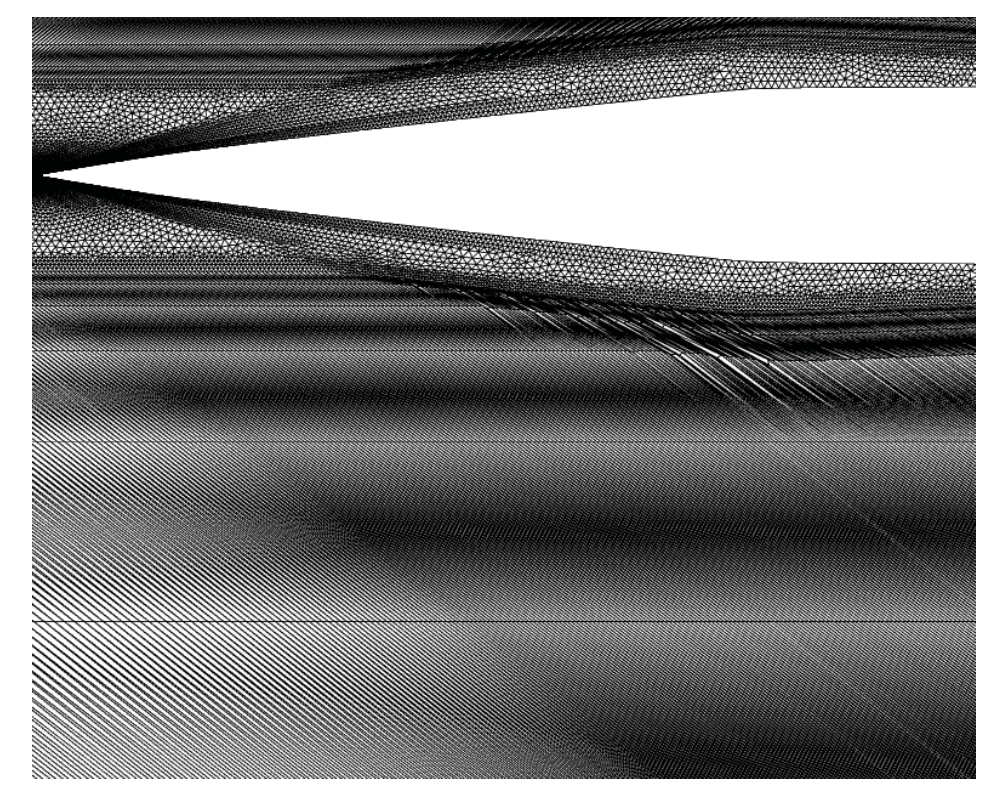

Figure 19. Final symmetry plane grid with cell shearing beginning at first collar layers. 


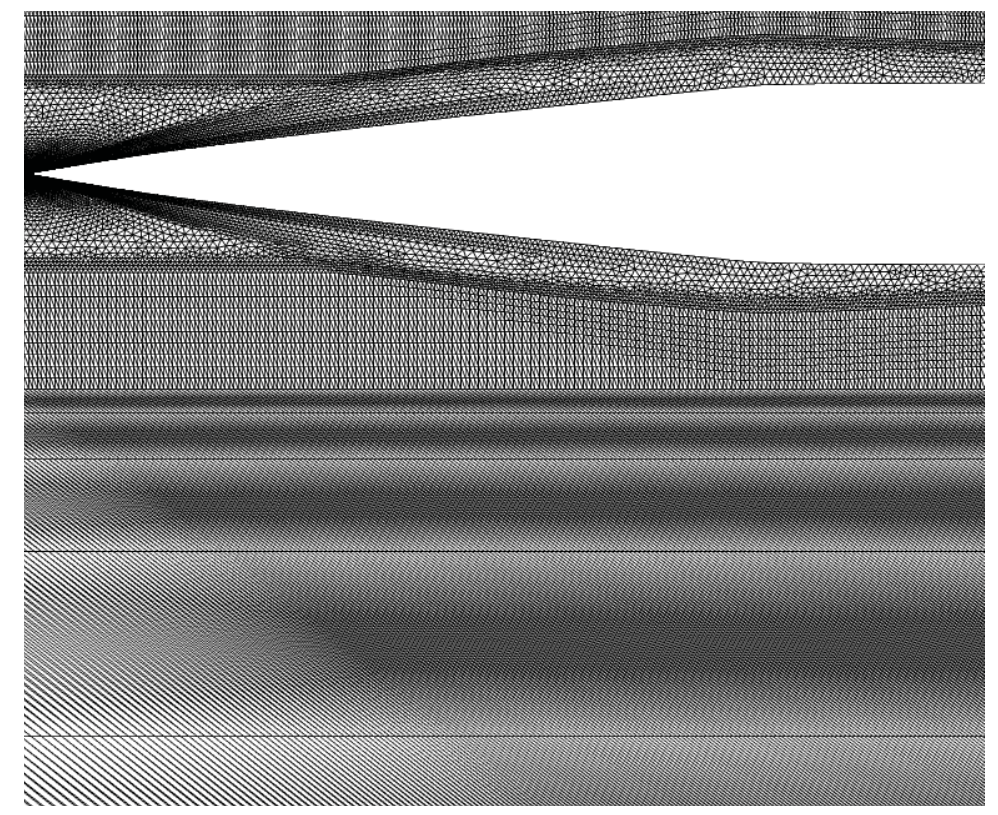

Figure 20. Final symmetry plane grid with cell shearing beginning after 10 collar grid layers.

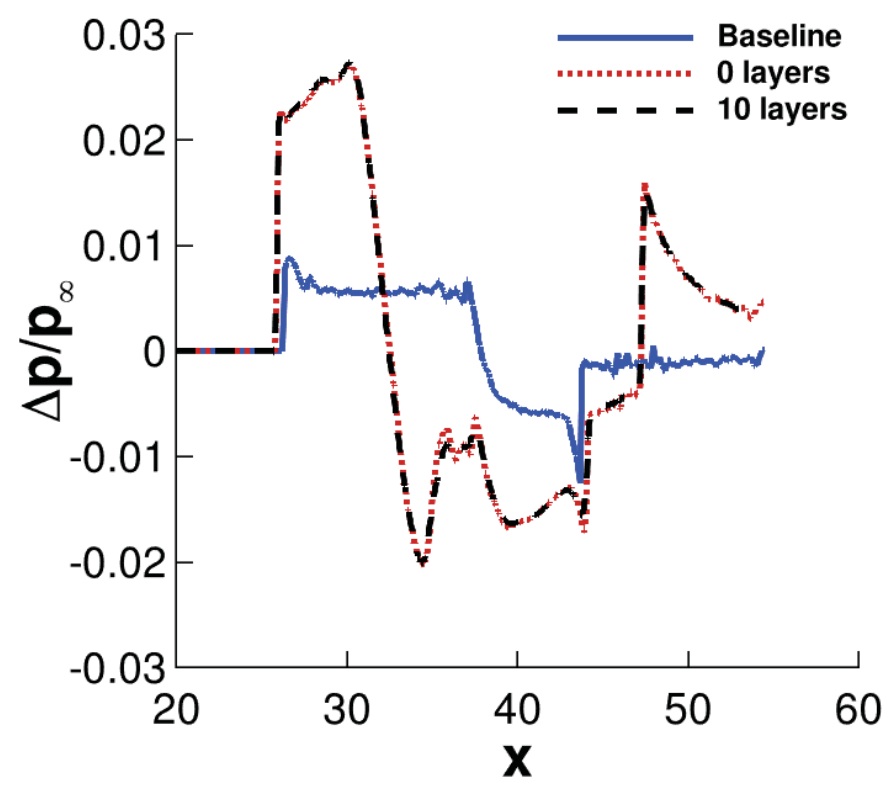

Figure 21. Effect of using initial un-stretched collar grid cell layers to absorb geometry modifications on the on-track signature at $\mathrm{H} / \mathrm{L}=1.2$ below the nose of the SEEB configuration. ( $x$ in inches, origin at the nose) 


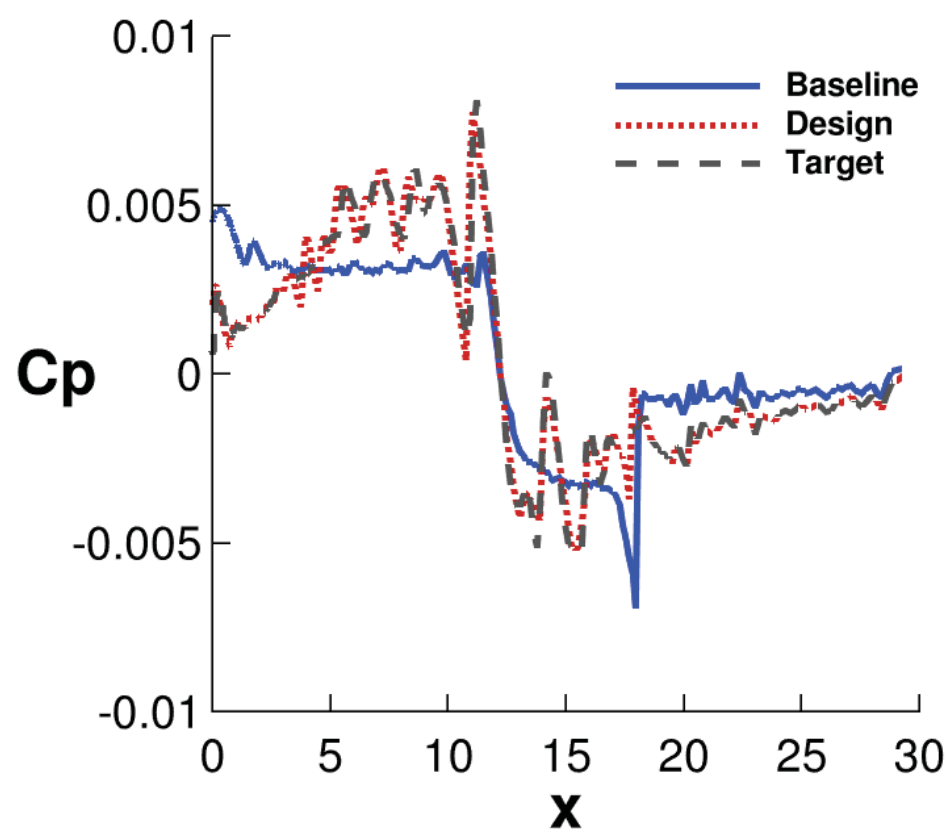

Figure 22. Pressure distributions for SEEB design to LM1021-based target pressure. ( $x$ in inches)

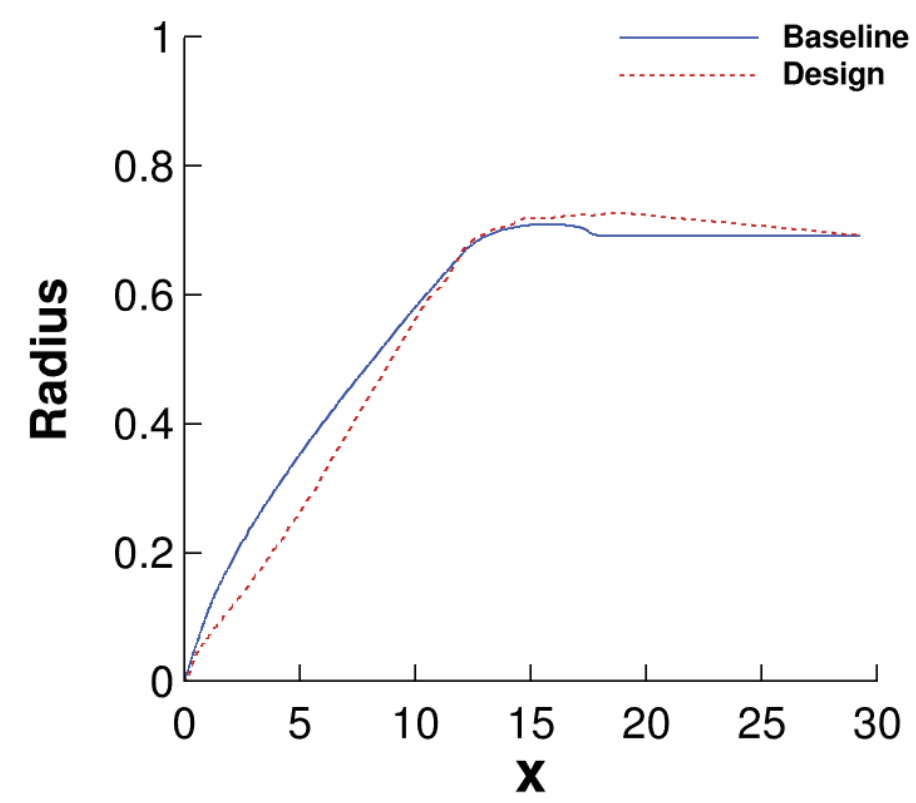

Figure 23. Axial distributions of radius for SEEB design to LM1021-based target pressure. ( $\mathrm{x}$ and radius in inches) 